\title{
LA EVOLUCION RECIENTE DE LA FECUNDIDAD EN ESPAÑA
}

I. Agüero y A. Olano Rey

La coyuntura demográfica española, en su lento discurrir, no deparaba a sus observadores sobresaltos ni rupturas espectaculares, hasta un pasado muy reciente. Pues bien, los últimos datos facilitados por el Instituto $\mathrm{Na}$ cional de Estadística sugieren, dentro de su provisionalidad, una aceleración súbita en la caída de todos los indicadores de natalidad y fecundidad durante los tres últimos años. Dicha aceleración arranca desde $1975-1976$ y se engarza en la caída lenta que venía observándose desde 1964, como tendremos oca. sión de precisar.

\section{La coyuntura reciente de la fecundidad española}

Los rasgos de esta coyuntura más reciente son los siguientes:

La cifra provisional de nacimientos para 1979 implica una tasa de natalidad del orden del 16,1 por 1.000 , frente al 18,1 por 1.000 observado en 1977, según la cifra definitiva de nacimientos, es decir, que la tasa de natalidad habría perdido dos puntos en dos años. La evolución secular de la tasa de natalidad se describe en el cuadro I. Cabe señalar que el valor provisional de la tasa de natalidad en 1979 sería el mínimo del siglo, inferior al mínimo anterior observado en 1939. 


\section{CUADRO I}

\section{Tasas de natalidad (1900-1979)}

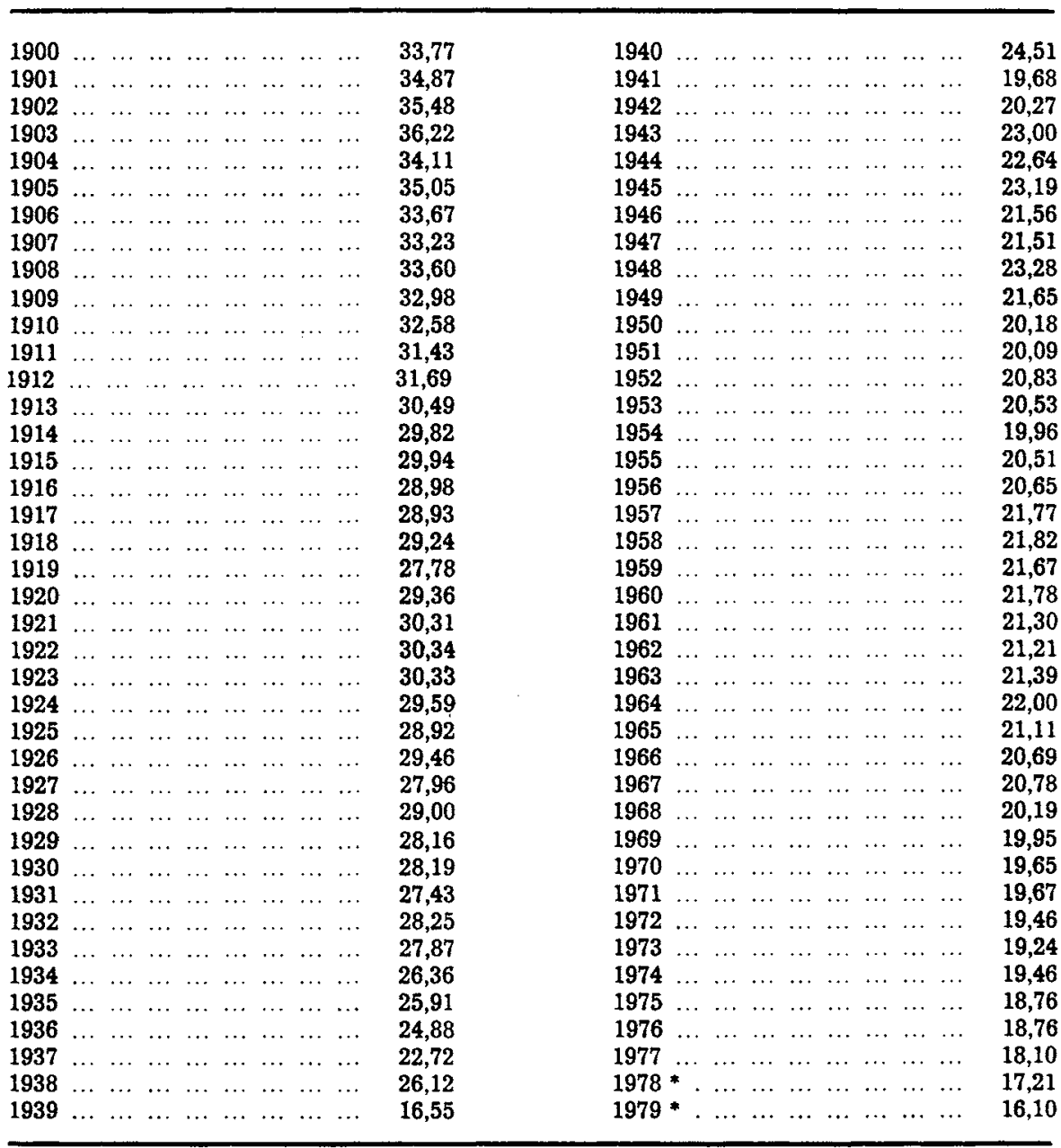

* Resultados provisionales.

FUENTE: INE.

Para situar el punto de partida de esta última caída acelerada de la serie de nacimientos y detectar la posible existencia de ciclos en su evolución, se han aplicado diversos tratamientos estadísticos de desestacionalización, los cuales implican siempre una cierta arbitrariedad y cuyos resultados se pres$\tan$ a interpretaciones diferentes. 
Por una parte, en el gráfico 1 se representa la serie mensual de nacimientos desde 1960 a 1978, desestacionalizada mediante el programa X-11 de la Oficina del Censo norteamericana; se observa que desde finales de 1972 la serie ciclo-tendencia resultante es claramente decreciente, aunque no se detecta ningún ciclo en la evolución. De la observación del gráfico 2, donde el número de nacidos de cada mes se ha sumado al número de nacidos en los once meses anteriores al mismo, se deduce que la cifra anual de nacimientos acelera intensamente su caída desde principios de 1977.

\section{GRAFICO NUM. 1}

\section{Nacimientos}
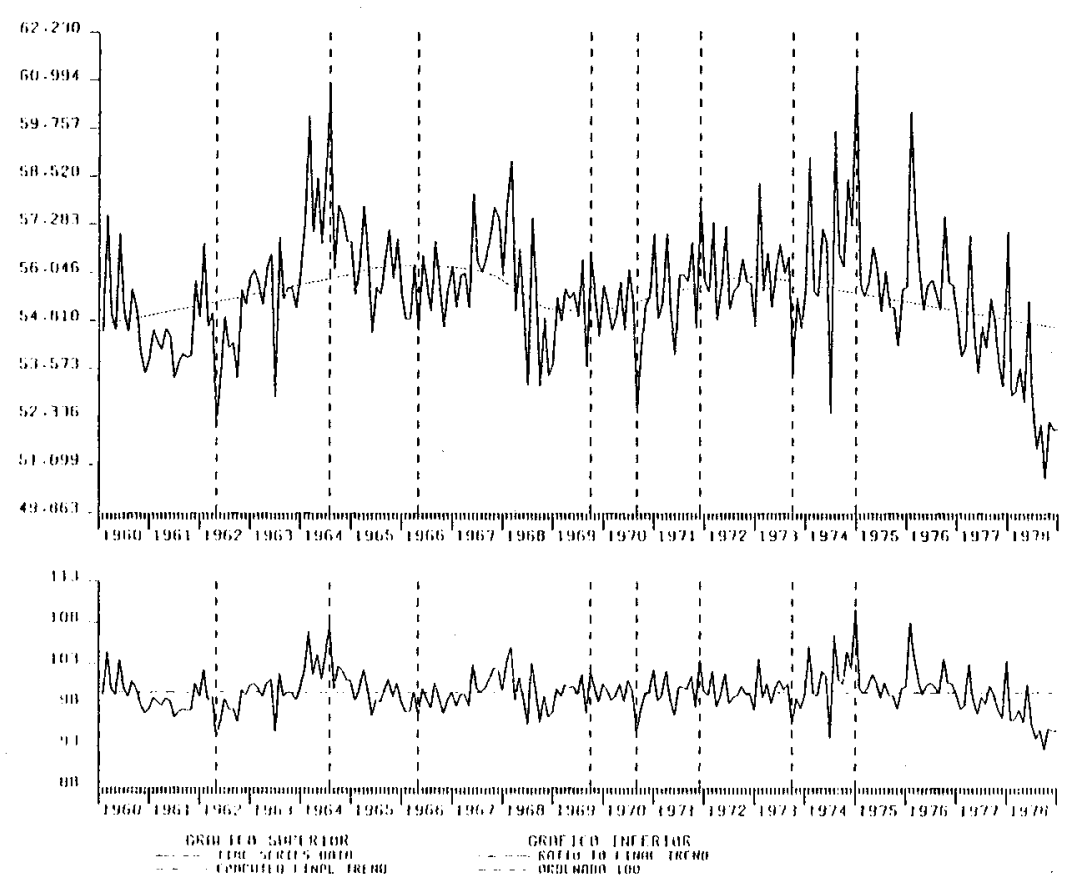

Utilizando un indicador más depurado de fecundidad, la tasa bruta de reproducción, podemos constatar asimismo una caída abrupta de la fecundidad que arranca desde 1974 y se acentúa desde 1977, tal como evidencia el gráfico 3. La tasa bruta de reproducción (TBR) representa el número de hijos que tendría una mujer sin riesgo de mortalidad, sometida a las tasas específicas de fecundidad observadas un año dado en cada grupo de edad de mujeres en período de vida fecunda. En el cuadro II correspondiente al gráfico 3 se representa la evolución de dicho indicador desde 1922 a 1979 . El último dato real es el de 1977, los valores de 1978 y 1979 se han estimado 
calculando las tasas específicas de fecundidad que, siendo proporcionales a las del año 1977 (hipótesis de calendario constante), al ser aplicadas a los efectivos de mujeres en cada grupo de edad fecunda en 1978 y 1979, dieran como resultado el número (provisional) de nacimientos registrados en dichos años ${ }^{1}$. En el año 1978 la TBR se mantiene por encima de las obtenidas en $1939,1941,1951$ y 1954, pero la tasa de 1979 sólo supera a la del año 1939.

\section{GRAFICO NUM. 2}

\section{Nacimientos anuales acumulados mensualmente}

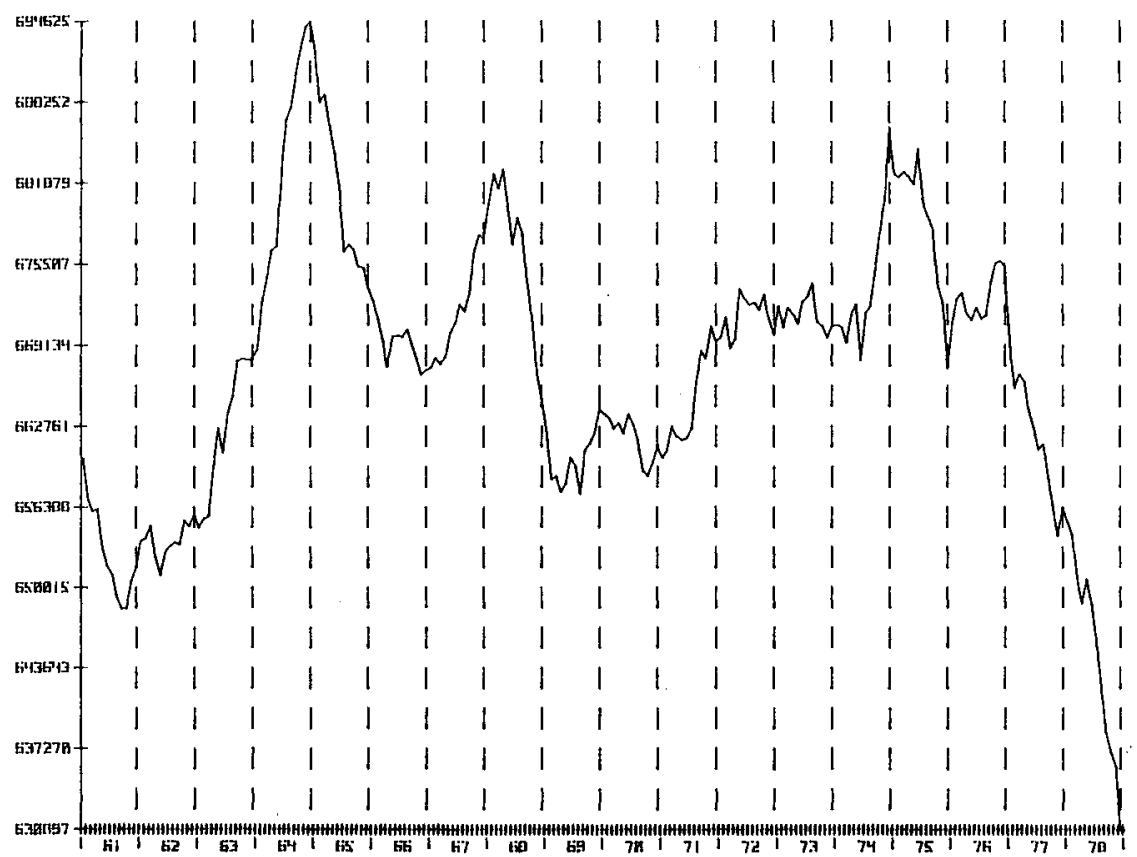

Para medir la intensidad de esta caída, se ha representado en el gráfico 4 la TBR tomando como índice 1.000 su valor máximo durante las últimas dácadas, observado en 1964. En este gráfico se pone de manifiesto aún más claramente que en el gráfico anterior cómo la caída a partir de 1975-76

'Por construcción: $N^{1977}=\sum_{u=15.19}^{45-49} \cdot{ }_{\varphi_{u}}^{1977} \cdot P_{u}^{1977}$

la estimación para 1978 resulta de la expresión:

$$
\mathrm{N}^{1978}={\underset{u=13-19}{45-49}}_{2} \cdot \mathrm{K} \cdot{ }_{\varphi_{u}}^{1977} \cdot P_{u}^{1978}
$$

Para 1979 el procedimiento ha sido semejante. 
es mucho más pronunciada que la del período 1964-1975. Esta precipitación en la caída de la fecundidad se detecta también a través de los índices de variación interanual de la tasa bruta de reproducción que se recogen en el cuadro III y el gráfico 5. Hay que subrayar que el último dato real, el de 1977 , presenta ya una disminución de -52 por 1.000 , y que, por tanto, las variaciones negativas que implican las cifras provisionales de los dos últimos años son bastante plausibles. Por otra parte, esta caída de la fecundidad no ha sido homogénea según los diferentes grupos de edad de mujeres en edad fecunda, sino que se ha acompañado de una transformación del calendario, en el sentido de una concentración de los nacimientos en las edades más jóvenes de la madre. Tomando la variación relativa de las últimas tasas específicas de fecundidad reales, las de 1977 , respecto al año de más alta fecundidad en los años setenta, es decir, 1974, se constata (cuadro III-B) que

\section{CUADRO II}

Tasa bruta de reproducción

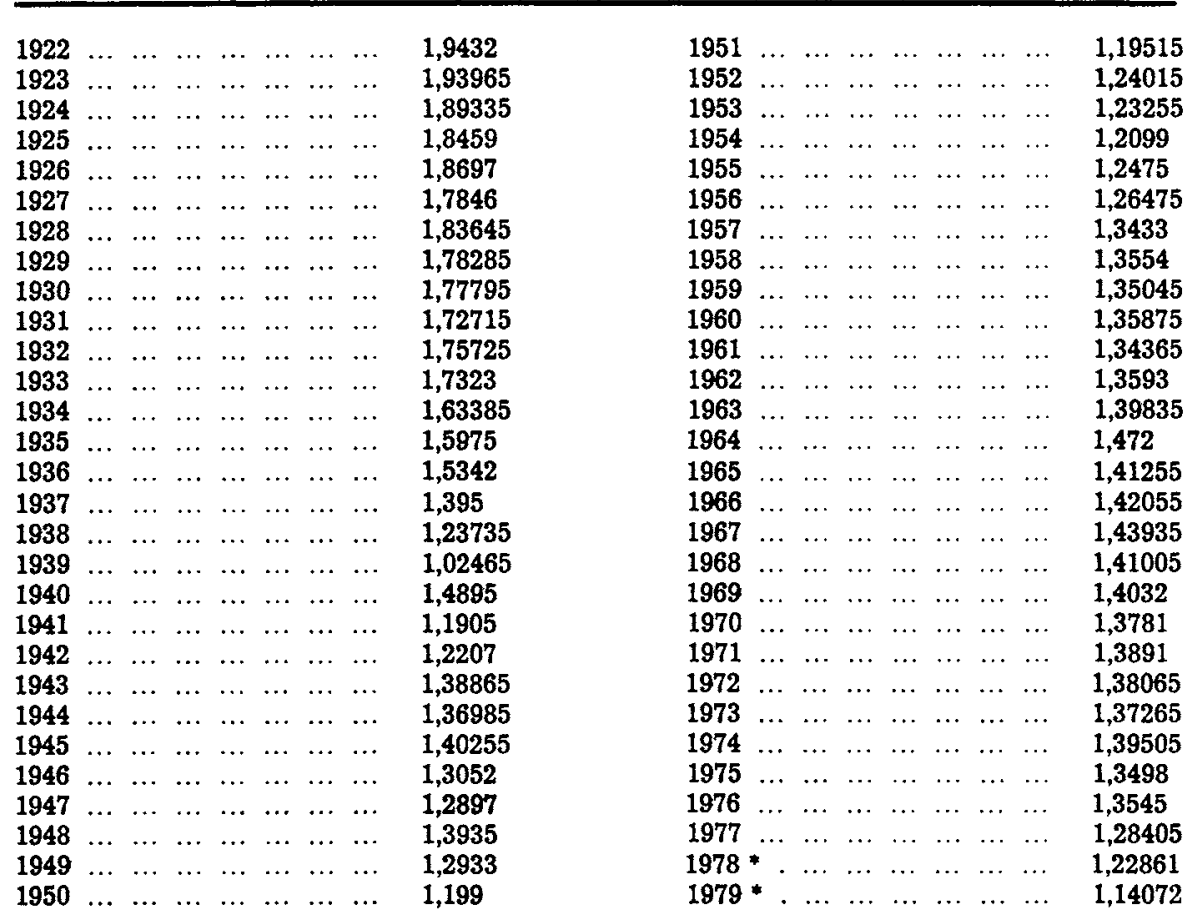

* Estimaciones.

FUENTE: INE. 
si bien la fecundidad global medida por la tasa bruta de reproducción ha disminuido en un 79 por 1.000 , ha aumentado, sin embargo, la fecundidad de las mujeres de quince a diecinueve años en un 227 por 1.000 , mientras que la disminución es creciente con la edad desde un 15 por 1.000 en las mujeres de veinte a veinticuatro años hasta un 184 por 1.000 entre las de cuarenta a cuarenta y cuatro años y un 132 por 1.000 entre las de cuarenta y cinco a cuarenta y nueve años. Como se suele constatar también longitudinalmente, la disminución de la fecundidad se ha acompañado en un rejuvenecimiento del calendario.

\section{GRAFICO NUM. 3}

Tasa bruta de reproducción (1922-1979)

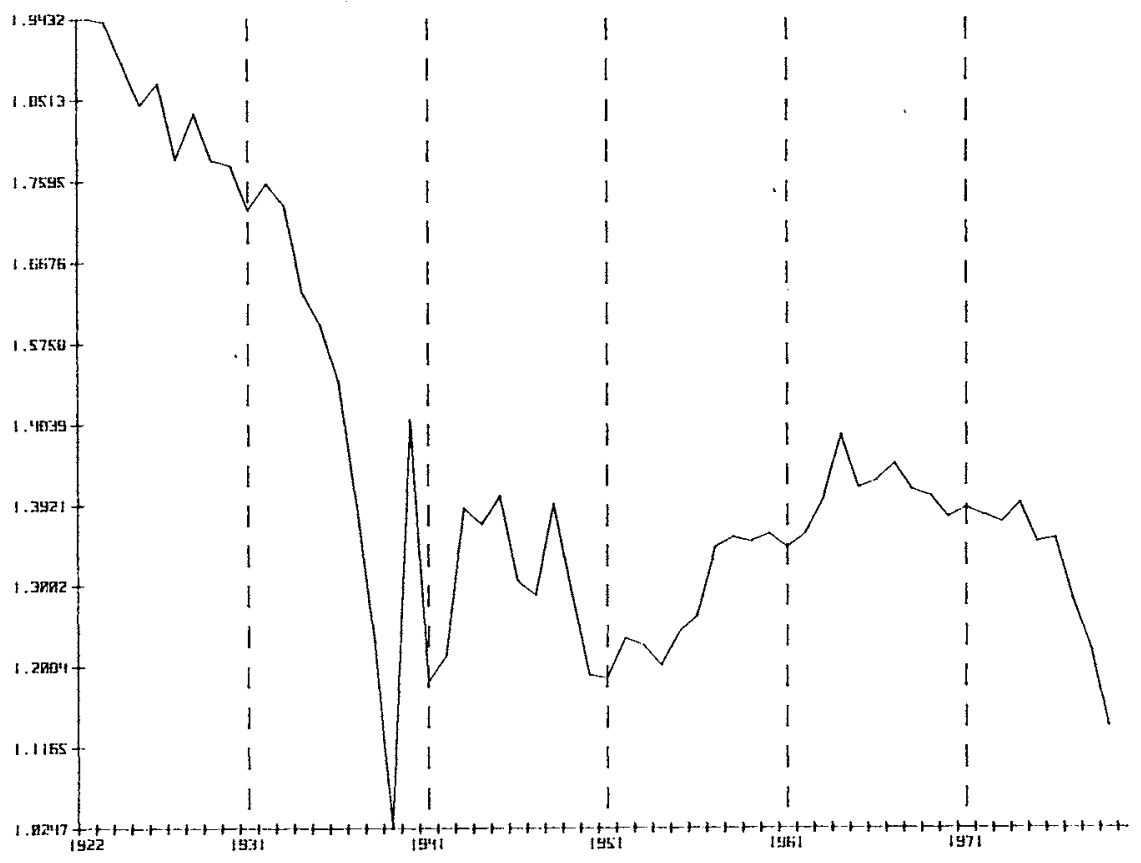

A un primer nivel de análisis cabe preguntarse si se trata de un accidente pasajero respecto a la evolución precedente o bien de un descenso prolongado con repercusiones decisivas en la evolución ulterior de la población española.

En demografía, la interpretación de una caída tan abrupta en los indicadores transversales (o cross-section) plantea una interrogación general bien conocida en análisis demográfico, a saber: se trata de una simple modificación en el calendario del fenómeno de la fecundidad o de una variación en la intensidad de dicho fenómeno, es decir, de una disminución de la descen- 
dencia final de las generaciones, que pasan actualmente por las edades de más alta fecundidad. Para dar una respuesta definitiva a dicha interrogación, habría que esperar la llegada de dichas generaciones al final de su vida fecunda, momento en el que el análisis longitudinal podría conjugarse con el transversal en la interpretación de nuestra situación demográfica de hoy.

Pero antes de que dicho análisis retrospectivo sea factible, la referencia al pasado reciente de la fecundidad española, así como la comparabilidad internacional y la desagregación provincial podrían explicarnos en buena medida las evoluciones demográficas en curso. Sin duda, todas las transformaciones ocurridas en el régimen demográfico español a lo largo de su trayectoria en el presente siglo, por no citar las mutaciones en sus determinantes socio-económicos, están incidiendo en la situación demográfica del momento.

Más allá de cualquier alarmismo respecto a los datos de estos dos últimos años, todavía provisionales, este artículo, en una primera aproximación al tema, intenta tanto como informar sobre la coyuntura demográfica más reciente, precisar en qué términos se plantea la interpretación de la evolución de la fecundidad en España durante las últimas décadas.

\section{GRAFICO NUM. 4}

Variación tasa bruta de reproducción $(1964=1.000)$

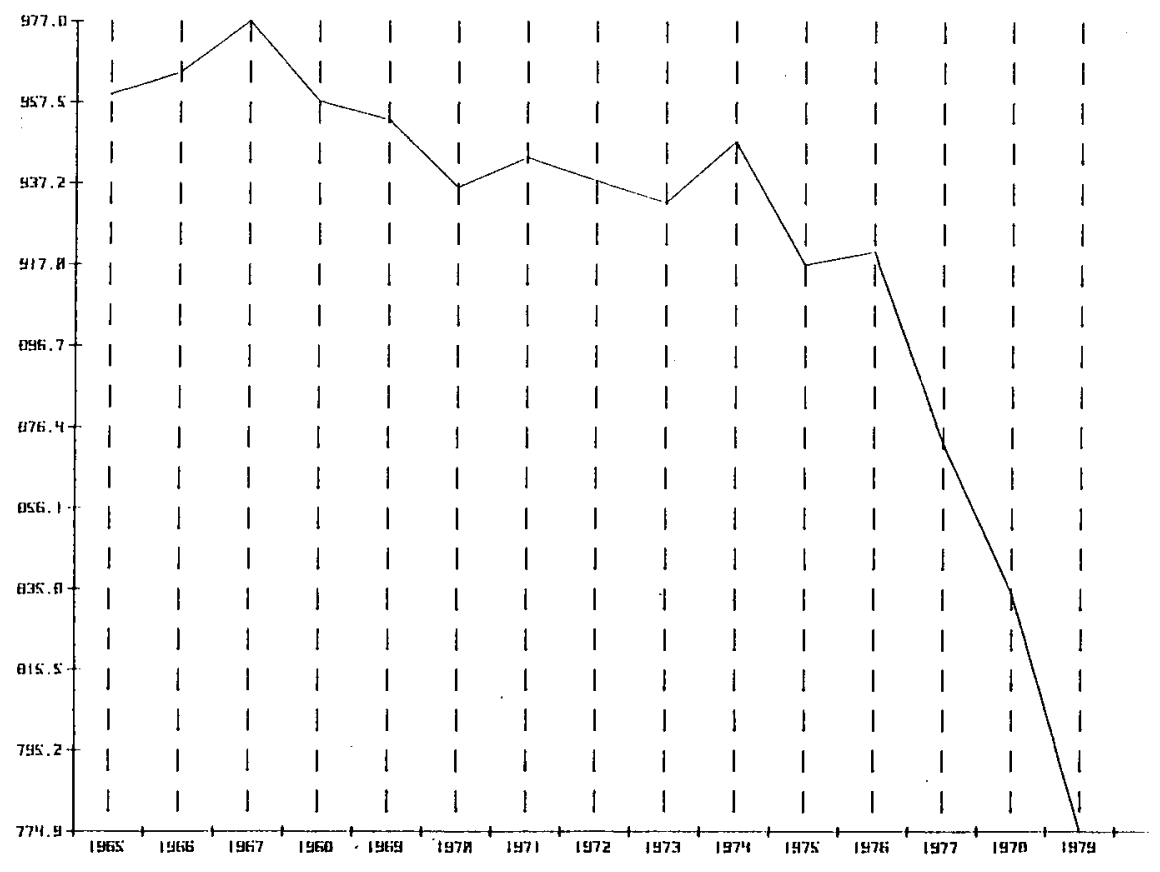




\section{CUADRO III}

Variación interanual de la tasa bruta de reproducción $(\% / 00)$

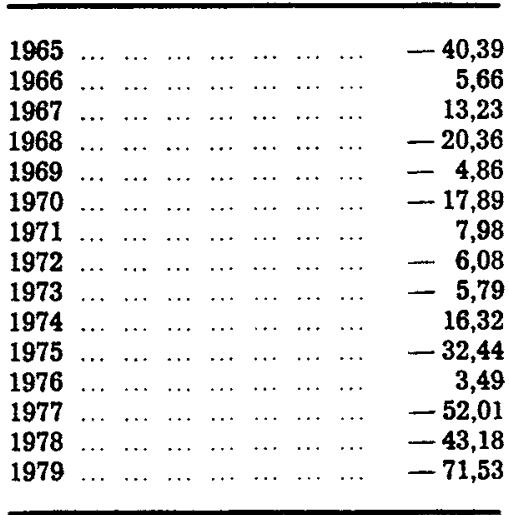

\section{CUADRO III B}

Evolución de las tasas específicas de fecundidad por grupos de edad durante el periodo 1974-1977

\begin{tabular}{lcccccccc}
\hline & $15-19$ & $20-24$ & $25-29$ & $30-34$ & $35-39$ & $40-44$ & $45-49$ & $R=5 \Sigma$ \\
\hline 1974 & 0,01002 & 0,06727 & 0,09597 & 0,06148 & 0,03170 & 0,01159 & 0,00098 & 1,39505 \\
1977 & 0,01230 & 0,06626 & 0,08566 & 0,05465 & 0,02764 & 0,00945 & 0,00085 & 1,28405 \\
$\frac{1977}{1974} \times 1.000-1$ & +227 & -15 & -107 & -111 & -128 & -184 & -132 & -79 \\
\hline
\end{tabular}

\section{El contexto demográfico actual de la fecundidad} en los paises industriales

En primer lugar, ¿cómo se sitúa esta evolución demográfica española en su contexto internacional más próximo y afín, el de los países industrializados de Occidente, en lo que a la natalidad y la fecundidad se refiere?

El conjunto de los países industrializados de Occidente conoce actualmente una caída sostenida de la fecundidad, que comenzó hacia 1964. Según los datos del cuadro IV, «los movimientos recientes de las tasas brutas de natalidad para el conjunto de países de la OCDE presentan una similitud' sorprendente. Las tasas de natalidad han bajado respecto a sus niveles de principios de los años sesenta, en todos los países de la OCDE. Desde 1970-71 
estas tasas han acusado una caída rápida en la gran mayoría de los países. Sin embargo, en algunos casos las cifras más recientes manifiestan una interrupción al menos provisional de este movimiento a la baja» ${ }^{2}$, tal es el caso de los Estados Unidos. En 1978, último año para el que se dispone de cifras reales, las tasas de natalidad de la mayor parte de los países de Europa occidental permanecen a un nivel muy bajo, casi todos se sitúan por debajo del 13 por 1.000 , cota jamás alcanzada en tiempos de paz ${ }^{3}$.

Merece especial atención, por lo que puede suponer de signo anticipador para nuestro país, el caso de Italia, cuya tasa de natalidad ha perdido dos puntos y medio por mil en tres años.

\section{GRAFICO NUM. 5}

Variación interanual tasa bruta de reproducción

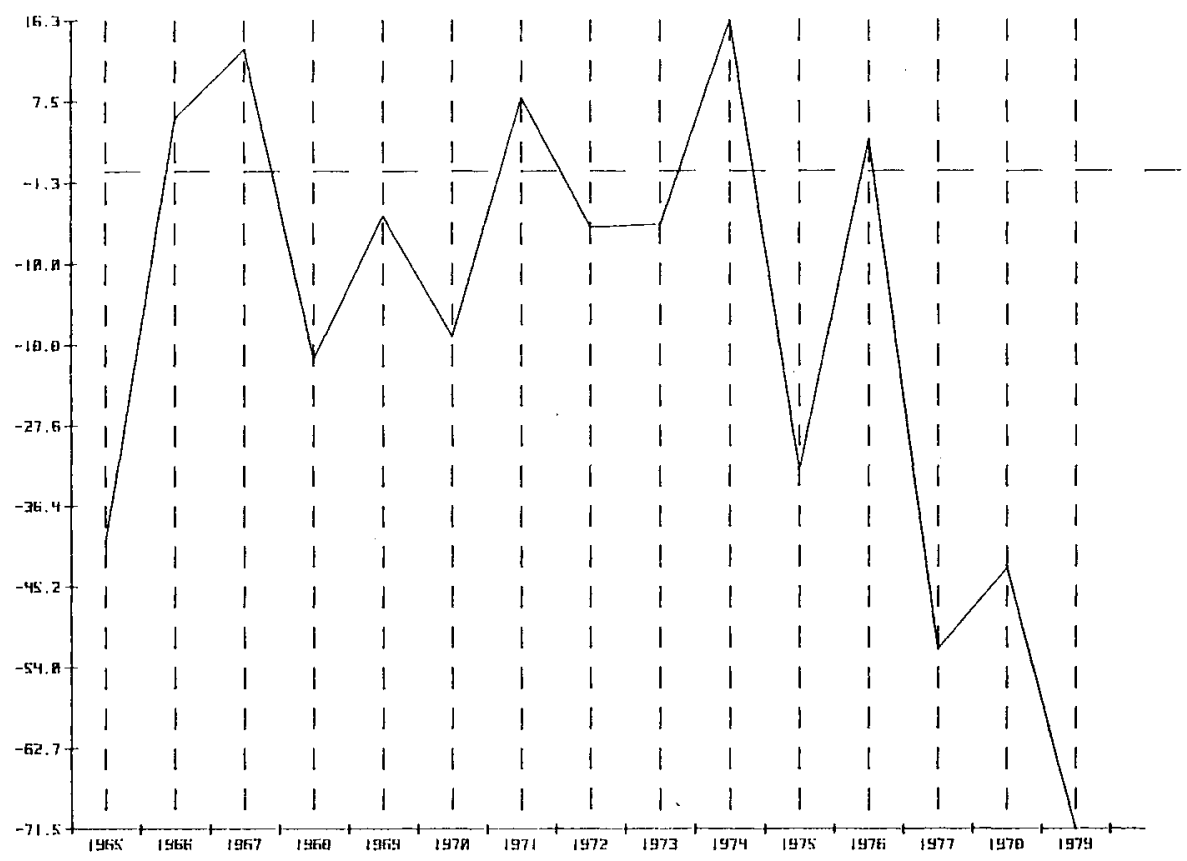

La natalidad española, cuyo valor máximo se sitúa en la primera mitad de los sesenta, a casi un punto por mil sobre la media de los países de la OCDE, presenta una evolución también decreciente. Sin embargo, su ritmo de caída es mucho más lento, de tal manera que en 1975 su nivel es supe-

2 OCDE, L'évolution démographique de 1950 à 1990, París, 1979, p. 7.

3 Cf. A. Monnier, "La conjoncture démographique: L'Europe et les pays développés d’outre-mer", Population, núms. 4-5, 1979, p. 886. 


\section{CUADRO IV}

Tasa de natalidad en los países de la OCDE

\begin{tabular}{|c|c|c|c|c|c|c|c|c|c|c|}
\hline & 1950 & 1960 & 1970 & 1975 & 1976 & 1977 & 1978 & 1980 & 1985 & 1990 \\
\hline $\begin{array}{llll}\text { Alemania } & \ldots & \ldots & \ldots\end{array}$ & 16,5 & 17,5 & 13,2 & 9,7 & 9,8 & 9,5 & 9,4 & 9,7 & 10,8 & 11,0 \\
\hline Australia $\ldots \begin{array}{lll} & \ldots & \ldots\end{array}$ & 23,3 & 22,4 & 20,6 & 16,9 & 16,7 & 16,1 & 15,7 & 15,3 & 16,9 & 16,5 \\
\hline Austria $\ldots \ldots \ldots \ldots$ & 15,6 & 17,9 & 15,1 & 12,5 & 11,6 & 11,4 & 11,3 & 13,3 & 14,1 & 14,1 \\
\hline Bélgica $\ldots \ldots \ldots \ldots$ & 16,6 & 17,0 & 14,6 & 12,1 & 12,3 & 12,4 & 12,4 & - & - & - \\
\hline Canadá $\ldots \ldots \ldots \ldots$ & 27,1 & 26,8 & 17,4 & 15,7 & 15,8 & 15,5 & 15,2 & 16,3 & 16,2 & 14,7 \\
\hline $\begin{array}{cccc}\text { Dinamarca } & . . & \ldots & \ldots\end{array}$ & 18,7 & 16,6 & 14,4 & 14,2 & 12,9 & 12,2 & 12,2 & 13,8 & 13,8 & 13,9 \\
\hline $\begin{array}{lllll}\text { España } & \ldots & \ldots & \ldots & \ldots\end{array}$ & 20,2 & 21,8 & 19,6 & 18,8 & 18,8 & 18,1 & - & 18,7 & 18,6 & 18,4 \\
\hline Estados Unidos . ... & 24,0 & 23,8 & 18,2 & 14,7 & 14,8 & 15,3 & 15,3 & 16,4 & 17,2 & 16,3 \\
\hline $\begin{array}{cccc}\text { Finlandia } & \ldots & \ldots & \ldots\end{array}$ & 24,5 * & 18,5 & 14,1 & 14,2 & 14,1 & 13,9 & 13,5 & 14,6 & 14,0 & 13,0 \\
\hline $\begin{array}{lllll}\text { Francia } & \ldots & \ldots & \ldots & \ldots\end{array}$ & 20,5 & 17,9 & 16,7 & 14,1 & 13,6 & 14,0 & 13,8 & 14,0 & 13,8 & 13,5 \\
\hline $\begin{array}{ccccc}\text { Grecia } & \ldots & \ldots & \ldots & \ldots\end{array}$ & 20,0 & 18,9 & 16,5 & 15,7 & 16,0 & 15,5 & - & - & - & - \\
\hline $\begin{array}{lllll}\text { Irlanda } & \ldots & \ldots & \ldots & \ldots\end{array}$ & 21,6 & 21,5 & 21,7 & 21,4 & 21,6 & 21,4 & - & $21,2^{b}$ & $21,0^{\circ}$ & - \\
\hline $\begin{array}{lllll}\text { Islandia } & . . & \ldots & \ldots & \ldots\end{array}$ & 27,1 & 27,9 & 19,6 & 20,2 & 19,5 & 17,8 & - & 22,0 & 22,5 & 21,9 \\
\hline $\begin{array}{lllll}\text { Italia } & \ldots & \ldots & \ldots & \ldots\end{array}$ & 19,4 & 18,4 & 17,1 & 15,1 & 13,9 & 13,2 & 12,6 & 14,7 & 15,9 & 15,6 \\
\hline Japón . ... ... ... ... & 28,2 & 17,2 & 19,3 & 17,2 & 16,3 & 15,5 & 15,1 & 16,6 & 14,5 & 14,1 \\
\hline $\begin{array}{lll}\text { Luxemburgo } & \ldots & \ldots\end{array}$ & 13,9 & 15,9 & 12,9 & 11,1 & 11,0 & 11,4 & 11,4 & 12,3 & 13,4 & 13,6 \\
\hline Noruega .. ... ... ... & 19,0 & 17,3 & 16,8 & 14,0 & 13,3 & 12,6 & 12,7 & 13,1 & 13,2 & 13,4 \\
\hline Nueva Zelanda. ... & 25,9 & 26,5 & 21,9 & 18,5 & 17,7 & 17,3 & 16,3 & 17,3 & 16,0 & 14,9 \\
\hline Países Bajos ... ... & 22,7 & 20,8 & 18,3 & 13,0 & 12,9 & 12,5 & 12,6 & 11,5 & 12,0 & 11,8 \\
\hline Portugal . ... ... ... & 24,3 & 23,6 & 19,2 & 19,1 & 19,0 & 18,4 & - & - & - & - \\
\hline Reino Unido $\ldots \ldots$ & 16,2 & 17,5 & 16,3 & 12,5 & 12,1 & 11,8 & 12,3 & 11,7 & 15,3 & 16,2 \\
\hline $\begin{array}{lllll}\text { Suecia } & \ldots & \ldots & \ldots & \ldots\end{array}$ & 16,4 & 13,6 & 13,7 & 12,7 & 12,0 & 11,6 & 11,2 & 13,0 & 12,7 & 12,9 \\
\hline 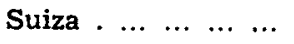 & 18,0 & 17,6 & 16,0 & 12,3 & 11,7 & 11,5 & 11,3 & 12,0 & 12,5 & 13,6 \\
\hline 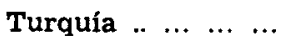 & 44,0 & 45,1 & 40,4 & 34,7 & - & - & - & 32,2 & 31,6 & 29,4 \\
\hline Yugoslavia $\ldots \ldots \ldots$ & 30,2 & 23,5 & 17,8 & 18,3 & 18,1 & 17,7 & 17,3 & 17,8 & 17,8 & 17,8 \\
\hline $\begin{array}{lllll}\text { Media } & \ldots & \ldots & \ldots & \ldots\end{array}$ & 22,1 & 21,0 & 18,0 & 15,9 & - & - & - & - & - & - \\
\hline 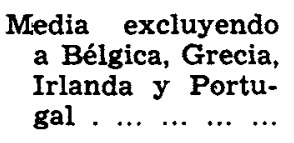 & - & - & - & 15,7 & - & - & - & 15,5 & 15,8 & 15,6 \\
\hline
\end{tabular}

- Nacimientos en Finlandia de sólo finlandeses; el denominador comprende a los extranjeros residentes en Finlandia.

- Para los años 1976-1981.

c Para los años 1981-1986.

FUENTE: OCDE, évolution démographique de 1950 à 1990, Paris, 1979. Para 1976, 1977 y 1978, cf. A. MonnIER, "L'Europe et les pays développés d'outre-mer", Population, núms. 4-5, 1979, p. 886. A partir de 1980, estimaciones. 
rior casi en tres puntos por mil a la media de los países de la OCDE. Esta caída lenta se prosigue hasta 1977; en dicho año la tasa de natalidad española es todavía del orden del 18,1 por 1.000 ; ya hemos comentado la caída abrupta en que se traducirían las provisionales de nacimientos en 1978 y 1979, en términos de dicha tasa de natalidad.

En conclusión sobre este punto hay que dejar muy en claro que el nivel de la natalidad española en 1977 era muy alto, sólo superado por Irlanda y Portugal, y probablemente por Turquía, en el conjunto de los países de la OCDE, y que una tasa del 16,1 por 1.000 en 1979 , si las cifras provisionales de nacimientos se confirman, es todavía alta respecto a los niveles de fecundidad europea e incluso respecto al área europea mediterránea.

La tasa bruta de natalidad no es un indicador adecuado para comparar los niveles de fecundidad entre diferentes países, pues está influenciado por el peso del efectivo de mujeres en edad fecunda y su estructura por edades, variables de una población a otra. Por esta razón se presenta en el cuadro $\mathrm{V}$ la evolución de otro indicador más depurado, capaz de medir la fecundidad en estado puro, a saber: el número medio de hijos por mujer o índice transversal de fecundidad, para el conjunto de los países de la OCDE.

La caída de la fecundidad que se inicia simultáneamente en el conjunto de los países occidentales en 1965, incluida España, siguiendo una sincronía tan sorprendente como inexplicable, se prosigue durante todo el período. Sin embargo, los ritmos, aceleraciones y suelos varían en el tiempo según los países: la disminución ha sido precoz en Alemania Occidental, en Estados Unidos, en el Reino Unido; la caída rápida no se manifiesta en Francia hasta 1973.74 y en Italia hasta 1975-77, mientras que se alcanzan ciertos suelos en 1970 en Estados Unidos, en 1971 en Francia, Irlanda... ${ }^{4}$. Los datos disponibles, con frecuencia todavía estimaciones, para 1978, incitan a pensar que hay una ralentización de la caída. Sin embargo, sería prematuro hablar hoy día de inversión de tendencia, incluso, simplemente de final de la caída ${ }^{5}$.

En este contexto podríamos caracterizar la evolución de la fecundidad española durante este período, por su sincronía en la evolución con el resto de los países occidentales y, sobre todo, por su tendencia a la homologación con los países más evolucionados demográficamente, si bien hay que precisar que el ritmo de caída de la fecundidad española hasta 1976 es más suave que en estos últimos países y que España se integra siempre con mayor proximidad en el área europea mediterránea.

Respecto al nivel de la fecundidad observado en 1976 en el conjunto de los países de la OCDE hay que observar que España tiene la fecundidad más alta después de Irlanda, con un promedio de hijos por mujer del orden

' J. N. Biraben, "L'Europe: Données statistiques", Population, núms. 4-5, 1978, páginas $992-993$.

5 A. Monnier, "La conjoncture démographique: L'Europe et les pays développés d'outre mer", Population, núms. 4-5, 1979, p. 887. 
del 2,6. Con el nivel actual de mortalidad alcanzado en estos países haría falta un promedio de 2,1 hijos por mujer para asegurar el reemplazamiento de las generaciones. La gran mayoría de los países en cuestión se sitúan por debajo de dicho nivel. Por último, cabe señalar que la tasa bruta de reproducción en 1979, tal como se ha estimado en el cuadro II, implica un promedio de 2,3 hijos por mujer, es decir, que el reemplazamiento de las generaciones sigue asegurado y mucho más el crecimiento de la población y que el nivel de la fecundidad española es todavía alto respecto al conjunto de los países occidentales más evolucionados demográficamente con los que tiende a homologarse. Sin embargo, si esta tendencia persiste al ritmo observado en los tres últimos años, y que se refleja en la pendiente de la curva de la tasa bruta de reproducción (ver gráfico 3), cabría anticipar una continuación en la caída de la fecundidad por debajo del nivel necesario al reemplazamiento de las generaciones, según ha preconizado Armando Sáez ${ }^{6}$ para la primera mitad de los años ochenta.

\section{Hacia una interpretación de la caida de la fecundidad: ¿tendencia secular o fluctuación?}

Si lo accidentado de la presente coyuntura demográfica pudiera resultar algo novedoso en el caso español, cabe recordar que la fecundidad de los países desarrollados ha conocido, desde hace casi un siglo, toda una serie de sobresaltos. El movimiento a la baja que se inicia en estos países simultáneamente hacia 1964 tan sólo sorprende o a veces inquieta por la profundidad de la caída.

Ahora bien, ¿qué explicación dan los demógrafos de esta caída de la fecundidad? La demografía es una ciencia joven que ha consagrado más del ochenta por ciento de su esfuerzo de investigación a la medida de los fenómenos y cuya capacidad hermenéutica o de interpretación de dichos fenómenos demográficos presenta todavía un alcance limitado, consecuencia de la imprecisión de sus marcos teóricos y sus instrumentos conceptuales.

Entre las corrientes teóricas que pretenden explicar los mecanismos que regulan la evolución de la fecundidad, podemos citar las teorías de inspiración microeconómica de la Escuela de Chicago (Becker y Mincer, y en general la «nueva economía doméstica»), según las cuales, un hijo es un bien de consumo duradero y el comportamiento de procreación de las parejas se explica en términos de cálculo económico coste/beneficio.

Una segunda corriente se refiere, sobre todo, a las transformaciones de las funciones del matrimonio y la familia. El postulado común a estas dos corrientes es que la función parental y, sobre todo, de procreación se ejerce

- A. SÁEz, "La fécondité en Espagne depuis le début du siècle", Population, número 6, 1979, p. 1014. 
cada día de una forma más deliberada y racional. Su limitación, también común, es que no aportan gran cosa a la comprensión de los movimientos de la fecundidad ${ }^{7}$.

\section{CUADRO V}

Número medio de bijos por mujer en los países de la $O C D E^{1}$

19651966196719681969197019711972197319741975197619771978

$\begin{array}{lllllllllllllllll}\text { Alemania } & \ldots & \ldots & 2,51 & 2,53 & 2,49 & 2,39 & 2,22 & 2,01 & 1,92 & 1,71 & 1,54 & 1,51 & 1,45 & 1,46 & 1,41 & 1,39\end{array}$

$\begin{array}{lllllllllllllllll}\text { Australia } & \ldots & \ldots & 2,97 & 2,88 & 2,85 & 2,89 & 2,88 & 2,85 & 2,94 & 2,74 & 2,49 & 2,40 & 2,23 & 2,14 & 2,09 & -\end{array}$ $\begin{array}{lllllllllllllllll}\text { Austria } & \ldots & \ldots & 2,68 & 2,66 & 2,63 & 2,59 & 2,50 & 2,32 & 2,20 & 2,10 & 1,95 & 1,93 & 1,84 & 1,70 & 1,61 & 1,61\end{array}$ $\begin{array}{lllllllllllllllll}\text { Bélgica } . & \ldots & \ldots & 2,61 & 2,52 & 2,42 & 2,31 & 2,25 & 2,25 & 2,21 & 2,09 & 1,96 & 1,83 & 1,74 & 1,75 & 1,75 & 1,75\end{array}$ $\begin{array}{llllllllllllllll}\text { Canadá } \ldots & \ldots & \ldots & 3,15 & 2,81 & 2,60 & 2,45 & 2,41 & 2,33 & 2,19 & 2,02 & 1,93 & 1,90 & 1,96 & 1,94 & 1,86\end{array}$ $\begin{array}{lllllllllllllllll}\text { Dinamarca } & . . & \ldots & 2,61 & 2,62 & 2,35 & 2,12 & 2,00 & 1,95 & 2,06 & 2,06 & 1,94 & 1,90 & 1,92 & 1,75 & 1,67 & 1,67\end{array}$ $\begin{array}{llllllllllllllllll}\text { España } & \ldots & \ldots & \ldots & 2,95 & 2,92 & 2,98 & 2,91 & 2,89 & 2,86 & 2,87 & 2,84 & 2,81 & 2,86 & 2,76 & 2,76 & 2,60 & -\end{array}$ $\begin{array}{lllllllllllllll}\text { Estados Unidos. } & 2,93 & 2,74 & 2,57 & 2,48 & 2,47 & 2,48 & 2,28 & 2,02 & 1,90 & 1,86 & 1,80 & 1,77 & 1,83 & -\end{array}$ $\begin{array}{lllllllllllllllll}\text { Finlandia } & \ldots & \ldots & 2,47 & 2,40 & 2,17 & 2,14 & 1,93 & 1,84 & 1,70 & 1,62 & 1,62 & 1,62 & 1,74 & 1,72 & 1,67 & 1,61\end{array}$ $\begin{array}{llllllllllllllllll}\text { Francia } & . . & \ldots & \ldots & 2,84 & 2,79 & 2,66 & 2,58 & 2,53 & 2,47 & 2,48 & 2,39 & 2,29 & 2,08 & 1,92 & 1,83 & 1,86 & 1,84\end{array}$ $\begin{array}{lllllllllllllllllll}\text { Grecia } & \ldots & \ldots & \ldots & 2,32 & - & - & - & - & 2,42 & 2,32 & 2,31 & 2,25 & 2,36 & 2,32 & 2,26 & -\end{array}$ $\begin{array}{llllllllllllllllll}\text { Irlanda } & \ldots & \ldots & \ldots & 4,06 & 3,95 & 3,84 & 3,77 & 3,83 & 3,84 & 3,96 & 3,88 & 3,78 & 3,74 & 3,57 & 3,50 & - & -\end{array}$

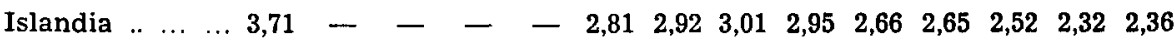
$\begin{array}{llllllllllllllllll}\text { Italia } & \ldots & \ldots & \ldots & 2,65 & 2,52 & 2,48 & 2,45 & 2,45 & 2,39 & 2,32 & 2,36 & 2,33 & 2,31 & 2,19 & 2,08 & 1,93 & -\end{array}$ $\begin{array}{llllllllllllllllll}\text { Japón } & \ldots & \ldots & \ldots & 2,14 & 1,60 & 2,22 & 2,13 & 2,23 & 2,13 & 2,16 & 2,14 & 2,14 & 2,05 & 1,91 & 1,86 & 1,81 & -\end{array}$ $\begin{array}{llllllllllllllll}\text { Luxemburgo. } & \ldots & 2,38 & 2,34 & 2,24 & 2,11 & 2,00 & 1,97 & 1,92 & 1,74 & 1,55 & 1,55 & 1,50 & 1,43 & 1,50 & 1,51\end{array}$ $\begin{array}{lllllllllllllllll}\text { Noruega } \ldots & \ldots & \ldots & 2,93 & 2,89 & 2,80 & 2,75 & 2,70 & 2,51 & 2,40 & 2,39 & 2,24 & 2,14 & 1,99 & 1,87 & 1,76 & 1,76\end{array}$

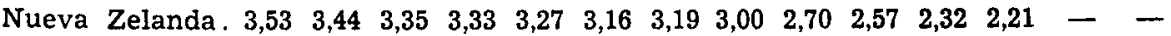
$\begin{array}{llllllllllllllll}\text { Países Bajos. } & 3,04 & 2,90 & 2,79 & 2,72 & 2,75 & 2,58 & 2,38 & 2,17 & 1,92 & 1,79 & 1,67 & 1,64 & 1,58 & 1,59\end{array}$

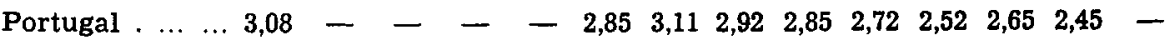
$\begin{array}{llllllllllllllll}\text { Reino Unido } & \ldots & 2,83 & 2,75 & 2,64 & 2,56 & 2,46 & 2,43 & 2,39 & 2,21 & 2,02 & 1,92 & 1,79 & 1,73 & 1,78 & 1,76\end{array}$ $\begin{array}{llllllllllllllllll}\text { Suecia } & \ldots & \ldots & \ldots & 2,42 & 2,36 & 2,28 & 2,07 & 1,93 & 1,92 & 1,97 & 1,91 & 1,87 & 1,89 & 1,78 & 1,69 & 1,65 & 1,59\end{array}$ $\begin{array}{llllllllllllllllll}\text { Suiza } . . . & \ldots & \ldots & 2,61 & 2,52 & 2,41 & 2,30 & 2,19 & 2,09 & 2,02 & 1,90 & 1,80 & 1,72 & 1,60 & 1,54 & 1,52 & 1,50\end{array}$ Turquía . ... ... - - - - - - -

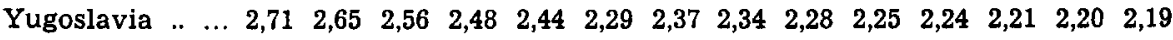

' Este indicador equivale a la tasa bruta de reproducción $(R)$ dividida por la proporción de nacimientos femeninos, es decir, aproximadamente: $S=\frac{R}{0,489}$

Fuente: Population, núms. 4-5, 1978, p. 994, y núms. 4-5, 1979, p. 886.

7 M. Fresel-Lozey, "Le colloque de Lille: la baisse de la fécondité. Limites et opportunité d'une action des pouvoirs publics", Population, núm. 6, noviembrediciembre, 1979 , p. 1103. 
Nos centraremos aquí en las teorías de enfoque más demográfico e histórico. Dentro de esta área más delimitada, podemos identificar tres tesis o interpretaciones de la evolución de la fecundidad a largo plazo: según la primera, se da una tendencia secular a la baja; para la segunda, la evolución de la fecundidad en los países industriales está sometida a fluctuaciones cíclicas; según la tercera, la última fase de la transición demográfica estaría también sometida a evolución cíclica. Simplificando, podríamos decir que estamos en presencia de dos tesis contrapuestas y una tercera integradora.

Para los defensores del modelo de la transición demográfica ${ }^{8}$ y para muchos otros autores que no se refieren explícitamente a ese modelo, la caída actual de la fecundidad prolonga su tendencia secular a la baja. El concepto de transición demográfica en sentido amplio, independientemente de diferencias respecto a su periodización en tres o cuatro fases y de las características atribuidas a su última fase, designa el paso, en el transcurso de un lapso más o menos largo de tiempo, desde un régimen tradicional de equilibrio demográfico caracterizado por tasas de mortalidad y natalidad muy altas e invariables, a un régimen moderno de equilibrio demográfico caracterizado por tasas de mortalidad y natalidad muy bajas y de nuevo invariables ${ }^{9}$. Pues bien, para los defensores de esta teoría demográfica, la disminución actual de la fecundidad anuncia la fase última de la transición demográfica, caracterizada por una natalidad y una mortalidad estables, de intensidad mínima, y en consecuencia, un crecimiento demográfico casi nulo. La objeción que habitualmente se formula frente a tal teoría es la subida de la natalidad (el célebre baby-boom) después de la segunda guerra mundial. ¿Se trata simplemente de un epifenómeno de una tendencia secular a la baja? ¿No se inscribe más bien en una línea de evolución marcada por fluctuaciones cíclicas del crecimiento demográfico? ${ }^{10}$.

Para una interpretación de la evolución de la fecundidad española, en términos de tendencia secular a la baja, según la cual la caída actual engarzaría con la tendencia decreciente que ciertamente se observa desde principios de siglo hasta la primera mitad de los años cincuenta, el gran escollo a superar y explicar es la subida de la fecundidad que perfila aparentemente casi un ciclo completo con un ramal al alza desde la segunda mitad de los años cincuenta hasta 1964 y un ramal a la baja desde 1965 hasta la repentina caída actual.

Ahora bien, esta especie de oscilación al alza confirmada por el análisis longitudinal en términos de descendencia final de las generaciones ${ }^{11}$, ¿sería

s Ch. F. Westofr, "Matrimonio y fertilidad en los países desarrollados", Scientific American Rew, edición española, febrero 1979, pp. 6-13.

- J. C. Chenars, "L'effet multiplicatif de la transition démographique", Population, núm. 6, noviembre-diciembre 1979 , p. 1138.

10 M. Fresel-Lozey, o. c. En este artículo se inspiran los apartados siguientes relativos a las tesis de Grauman y Easterlin.

11 A. SÁEz, o. c. 
un simple accidente histórico, o más bien habría que interpretarlo como un movimiento cíclico, dentro del nuevo régimen demográfico al que habría accedido la población española a partir de la segunda mitad de los años cincuenta?

La segunda escuela citada arranca de las ideas de John Grauman. A él se debe la idea de una relación entre la fecundidad de los padres y la de los hijos, que se traduciría en una relación negativa entre el efectivo de una generación y su fecundidad, a través de variables intermedias como la oferta de trabajo, o la situación económica relativa. En otros términos existe una relación entre fecundidad y mercado de trabajo: muy esquemáticamente, las dificultades que encuentran las generaciones numerosas para entrar en el mercado de trabajo les incitarían a reducir su fecundidad; las generaciones siguientes, menos numerosas, al no conocer dificultades de empleo, adoptarían un comportamiento menos maltusiano que se traduciría en un aumento de la fecundidad.

R. Easterlin, retomando el modelo de J. Grauman, propone una formulación que integra las aspiraciones y las necesidades de los individuos: éstas están, según él, ampliamente determinadas por las condiciones de vida que han conocido las generaciones jóvenes en sus familias de origen; en este sentido, su propensión a procrear será tanto más fuerte cuanto más comparable o ventajosa sea su situación respecto de las generaciones anteriores. A esta formulación económica, Easterlin ha sustituido más recientemente una expresión más demográfica, proponiendo como indicador de las fluctuaciones de la fecundidad la relación entre la población masculina de 30.64 años y la población masculina de $15-29$ años ${ }^{12}$. Durante este segundo período de edades no sólo se constituyen la mayor parte de los matrimonios y se producen la mayoría de los nacimientos de tales matrimonios, sino que también es la edad de inserción en el mercado de trabajo. Así, pues, un aumento de la relación entre los que tienen 35-64 años y los que tienen 15-34 años —una disminución de la relación inversa 15-29 años/30-64 años- sugeriría un mercado de trabajo poco saturado y, en consecuencia, un alza de la fecundidad, e inversamente.

Aplicado a la evolución demográfica de los países industrializados, el modelo de Easterlin reviste aparentemente un buen valor explicativo, pues pone de manifiesto, en efecto, un paralelismo entre la curva del indicador de estructura por edades y la del índice transversal de fecundidad. Es decir, que a una repartición por edades desfavorable (en el sentido del modelo) correspondería una fecundidad reducida, y viceversa. La caída de la fecundidad actual no correspondería, pues, a una caída secular, sino que se inscribiría más bien en un movimiento de oscilaciones sucesivas, determinadas por va-

12 R. EASTERLIN y otros, "Demographic influences on Economic Stability: The United States Experience", Population and Development Review, marzo 1878, páginas 1-22. 
riaciones de las estructuras por edad, producidas a su vez por fluctuaciones anteriores de la fecundidad.

A este respecto, dado que las proyecciones de población anticipan una mejora de la estructura por edades, según el modelo, en varios países: Estados Unidos ${ }^{13}$, Francia, Alemania, Inglaterra y País de Gales ${ }^{14}$, en la primera mitad de los ochenta podría producirse una subida en la natalidad o, al menos, una inversión de su tendencia a la baja actual.

\section{CUADRO VI}

Indice de estructura por edades

$\frac{\text { Varones } 15-34}{\text { Varones } 35-65}$

\begin{tabular}{|c|c|c|c|c|c|c|c|c|c|c|c|c|c|c|c|c|c|}
\hline 1900 & .. & $\ldots$ & & $\ldots$ & & & & 1,0429 & 1966 & & $\ldots$ & $\ldots$ & $\ldots$ & $\ldots$ & $\ldots$ & $\ldots$ & 0,8938 \\
\hline 1910 & $\ldots$ & $\ldots$ & $\ldots$ & $\ldots$ & $\ldots$ & $\ldots$ & $\ldots$ & 1,0618 & 1967 & & $\ldots$ & $\ldots$ & $\ldots$ & $\cdots$ & $\ldots$ & $\ldots$ & 0,8848 \\
\hline 1920 & .. & $\ldots$ & $\ldots$ & $\ldots$ & $\ldots$ & $\ldots$ & $\ldots$ & 1,1197 & 1968 & $\ldots$ & $\ldots$ & $\ldots$ & $\ldots$ & $\cdots$ & $\ldots$ & $\ldots$ & 0,8758 \\
\hline 1930 & $\ldots$ & $\ldots$ & $\ldots$ & $\ldots$ & $\ldots$ & $\ldots$ & $\ldots$ & 1,1941 & 1969 & $\ldots$ & $\ldots$ & $\ldots$ & $\ldots$ & $\ldots$ & $\ldots$ & $\ldots$ & 0,8526 \\
\hline 1940 & $\ldots$ & $\ldots$ & $\ldots$ & $\ldots$ & $\ldots$ & $\ldots$ & $\ldots$ & 1,1363 & 1970 & $\ldots$ & $\ldots$ & $\ldots$ & $\ldots$ & $\ldots$ & $\ldots$ & $\ldots$ & 0,8569 \\
\hline 1950 & $\ldots$ & $\ldots$ & $\ldots$ & $\ldots$ & $\ldots$ & $\ldots$ & $\ldots$ & 1,1302 & 1971 & $\ldots$ & $\ldots$ & $\ldots$ & $\ldots$ & $\ldots$ & $\ldots$ & $\ldots$ & 0,8630 \\
\hline 1960 & $\ldots$ & $\ldots$ & $\ldots$ & $\ldots$ & $\ldots$ & $\ldots$ & $\ldots$ & 1,0003 & 1972 & & $\ldots$ & $\ldots$ & $\ldots$ & $\ldots$ & $\ldots$ & $\ldots$ & 0,8756 \\
\hline 1961 & $\ldots$ & $\ldots$ & $\ldots$ & $\ldots$ & $\ldots$ & $\ldots$ & $\ldots$ & 0,9797 & 1973 & & $\ldots$ & $\ldots$ & $\ldots$ & $\ldots$ & $\ldots$ & $\ldots$ & 0,8983 \\
\hline 1962 & $\ldots$ & $\ldots$ & $\ldots$ & $\ldots$ & $\ldots$ & $\ldots$ & $\ldots$ & 0,9597 & 1974 & ... & $\ldots$ & $\ldots$ & $\ldots$ & $\ldots$ & $\ldots$ & $\ldots$ & 0,8948 \\
\hline 1963 & $\ldots$ & $\ldots$ & $\ldots$ & $\ldots$ & $\ldots$ & $\ldots$ & $\ldots$ & 0,9403 & 1975 & ... & $\ldots$ & $\ldots$ & $\ldots$ & $\ldots$ & $\ldots$ & $\ldots$ & 0,9095 \\
\hline 1964 & $\ldots$ & $\ldots$ & $\ldots$ & $\ldots$ & $\ldots$ & $\ldots$ & $\ldots$ & 0,9214 & 1976 & $\ldots$ & $\ldots$ & $\ldots$ & $\ldots$ & $\ldots$ & $\ldots$ & $\ldots$ & 0,9226 \\
\hline 1965 & $\cdots$ & $\ldots$ & $\cdots$ & $\ldots$ & $\ldots$ & $\cdots$ & $\ldots$ & 0,9030 & 1977 & & $\ldots$ & $\ldots$ & $\ldots$ & $\ldots$ & $\ldots$ & $\ldots$ & 0,9269 \\
\hline
\end{tabular}

Hasta 1960 la estructura por edades está tomada de los Censos de población. A partir de 1961 los datos se han tomado de la publicación "Población, Actividad y Ocupación en España (reconstrucción de las series históricas 1960-1978)". Ministerio de Economía, Madrid, 1979.

Para contrastar la hipótesis de Easterlin en el caso español, hemos tomado como índice de la estructura por edad la relación entre los varones de 15 a 34 años y los de 35 a 64 años, por considerarse que la edad media al contraer matrimonio en España es más elevada que en Estados Unidos. En el cuadro VI se recoge la evolución de esta relación; hay que notar que en las fechas anteriores a 1960 sólo se dispone de información sobre la estructura por edades en los años censales. La influencia de la fecundidad sobre la estructura por edad, unos 15 ó 20 años más tarde se observa claramente en nuestros datos, basta contrastar a este respecto el cuadro II y el cuadro VI. En efecto, el indice de estructura es decreciente desde 1930

${ }^{13}$ EASTERLIN, ibidem.

${ }_{14}$ J. Bourgeors-Pichat, "La baise actuelle de la fécondité en Europe s'inscritelle dans le modèle de la transition démographique", Population, núm. 2, 1979. 
a 1969, como consecuencia de la caída de la fecundidad hasta los años cincuenta, crece en el período 1970-1973, resultado del crecimiento de la natalidad en el período $1954-1958$ y aumenta de nuevo a partir de 1975 , reflejando el alza en la fecundidad de 1961 a 1964.

\section{GRAFICO NUM. 6}

\section{Tasas de natalidad (1900+1979)}

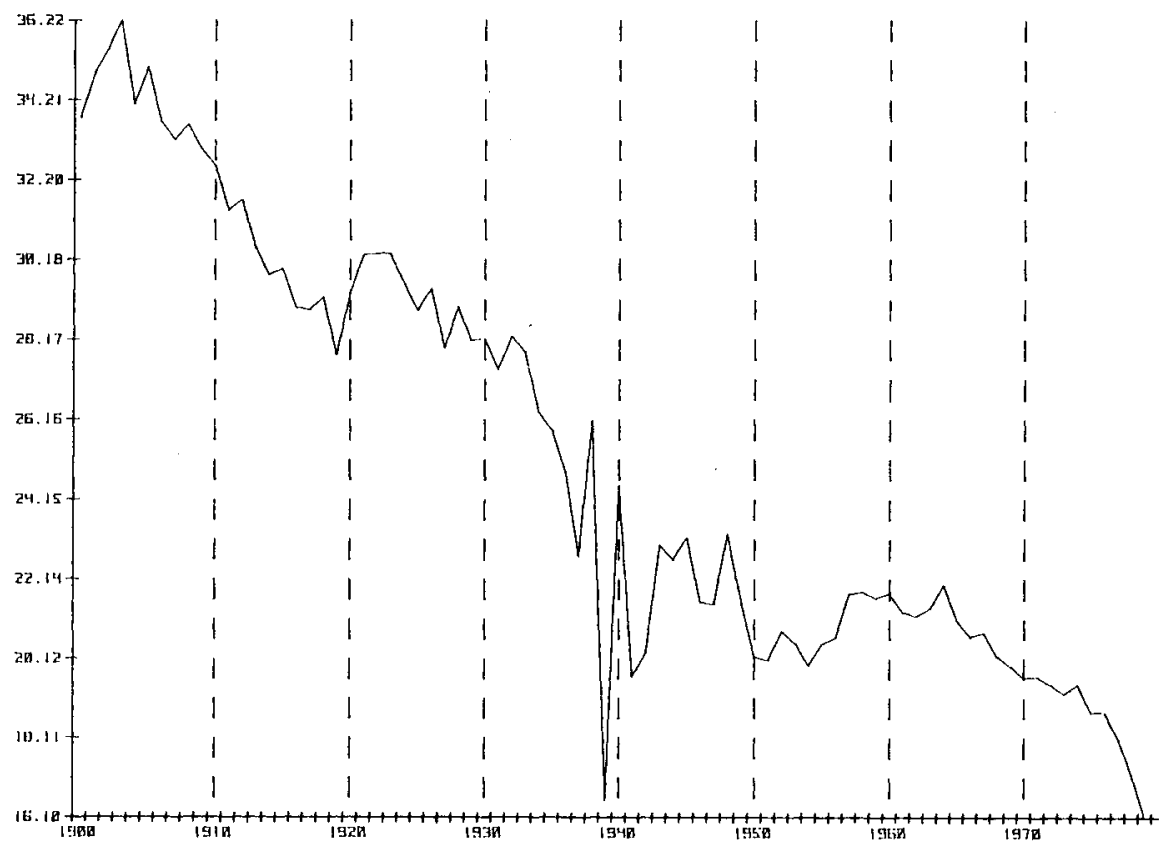

Sin embargo, no existe correlación entre la fecundidad actual y la estructura por edad actual durante el período analizado 1960-1975. En otras palabras, la tesis de Easterlin no se verifica en el caso español, en particular el descenso en la fecundidad a partir de 1964 se produce en un período en que el número de jóvenes relativo al de mayores de 35 años es decreciente, es decir, favorable y propenso a una mayor fecundidad según la lógica del modelo. El coeficiente de correlación calculado entre la tasa de natalidad y el índice de estructura por edad, ambas series estudiadas durante el período 1960-1975 es de 0,6639. Para el mismo período, el coeficiente de correlación entre la tasa bruta de reproducción y la estructura por edad es de $-0,3027$ (la correlación entre la tasa bruta de natalidad y la TBR es para 1960-1975 de 0,3887). 
El hecho de que en nuestro país no se verifique la hipótesis de Easterlin no descarta la posibilidad de una evolución cíclica de la fecundidad sin correlación con el índice de estructura por edad. En realidad la observación del gráfico 3 (TBR 22.79) sobre todo en el período 1954-1979, da pie para hacer esta conjetura. Para contrastar esta hipótesis se ajustó una curva de tendencia a la TBR y se calcularon las desviaciones con respecto a la misma para estudiar la existencia del ciclo. La limitación impuesta por el número de observaciones de la serie ( 55 en la fecha en que se realizó el análisis, es decir, el período 1922-1976), hizo inaplicables los programas estándar de análisis de series (X-11, Never). La función de tendencia utilizada fue de tipo exponencial, exactamente se ajustó la función $K(1+r)^{t}$ en cada punto, estimándose los parámetros $K$ y $r$ por el método de los mínimos cuadrados sobre un número prefijado de observaciones de fechas inmediatamente anteriores a la de referencia y a ella misma. El número de observaciones tomadas para realizar la estimación mínimo-cuadrática se hizo variar de cinco a veinticinco. En los gráficos 7.1 a 7.5 se han presentado los valores de $r$ obtenidos del ajuste sobre cinco, diez, quince, veinte y veinticinco observaciones, res-

\section{GRAFICO NUM. 7.1}

Tasa de crecimiento anual acumulativo media, estimada sobre intervalos de cinco observaciones

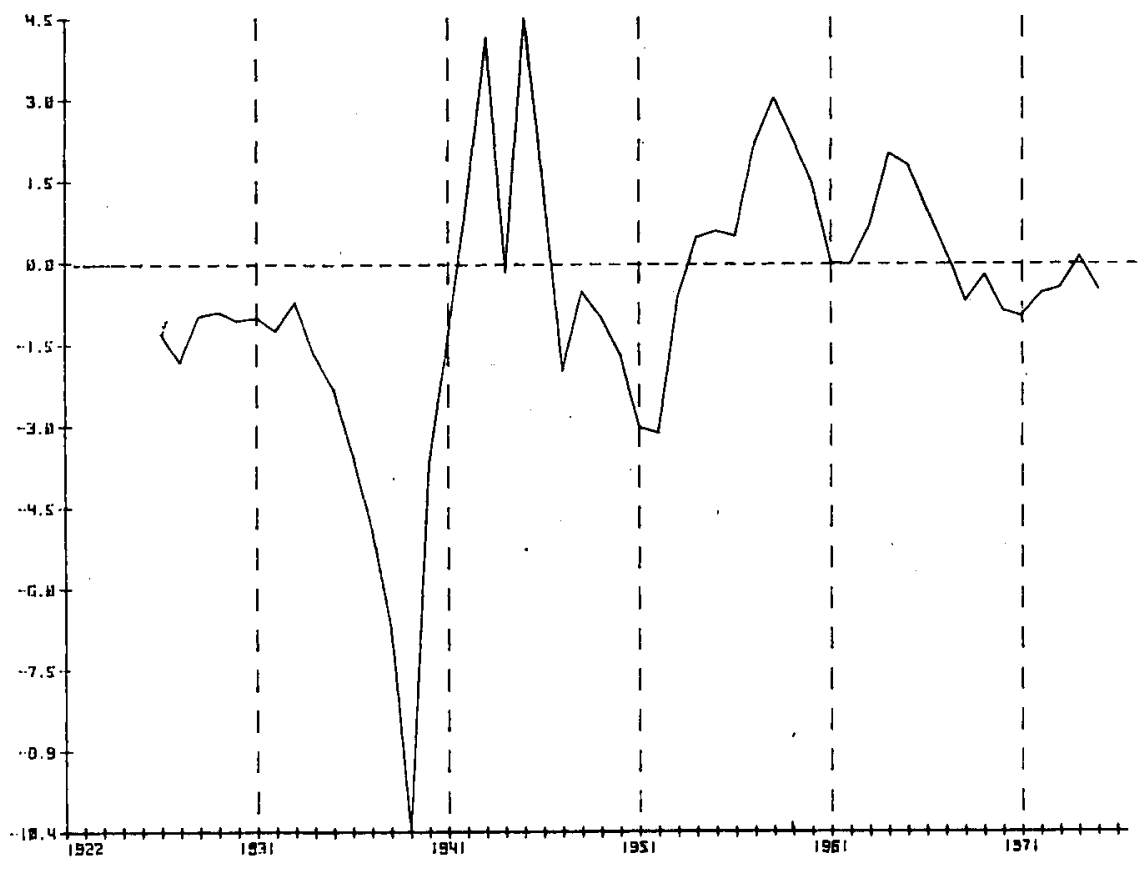


pectivamente. A partir de la fórmula de tendencia ajustada, $K(1+r)^{t}$, se deduce que $r$ representa la tasa de crecimiento anual acumulativa media del período de cinco, diez, quince, veinte o veinticinco años que finaliza en la fecha que corresponde en el eje de abscisas. La irregularidad de la serie original se refleja en las fluctuaciones en el valor de $r$ más acusadas lógicamente cuanto menor es el período sobre el que está calculado. En este sentido, los gráficos 7.4 y 7.5 muestran claramente que el decrecimiento de la TBR en el período 1922-1941 es mucho más pronunciado que el de los años posteriores a esa fecha, reflejándose este hecho en que $r$ (tasa de crecimiento medio anual acumulativa) es creciente en el gráfico 7.4 desde 1942 a 1969 y el 7.5 desde 1946 a 1974 y positiva en el intervalo $1962-1969$ y 1959 1974 , respectivamente.

\section{GRAFICO NUM. 7.2}

Tasa de crecimiento anual acumulativo media, estimada sobre intervalos de diez observaciones

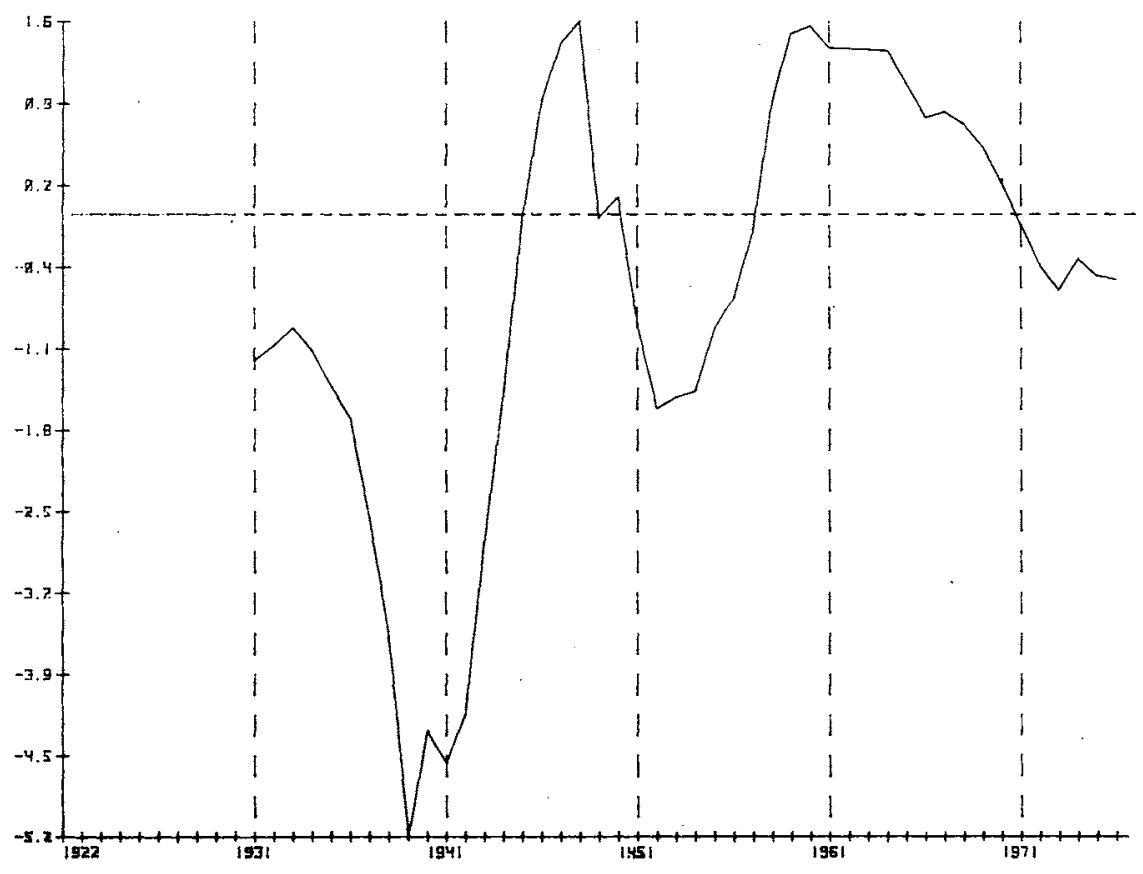

Esta comprobación pone de manifiesto que no se puede explicar la evolución de la fecundidad española desde principios de siglo en términos de una simple caída tendencial secular. Efectivamente, la evolución de la fecun- 
didad en España es decreciente desde 1900 hasta la primera mitad de los años cincuenta, medida en términos de descendencia final de las generaciones ${ }^{15}$; dicha «disminución secular anterior no había sufrido ninguna alteración incluso después de la guerra civil» ${ }^{16}$. A través de indicadores transversales (que son siempre reconstrucciones teóricas y artificiales) podríamos establecer una periodización diferente, pues la evolución de la curva de la tasa bruta de reproducción, por ejemplo, es mucho más accidentada.

El aumento de la fecundidad española desde mitad de los años cincuenta hasta 1964 es relativamente importante, según los indicadores transversales y longitudinales, es decir, que la subida se tradujo en un aumento de la intensidad del fenómeno: aumentó la descendencia final de las generaciones afectadas.

\section{GRAFICO NUM. 7.3}

Tasa de crecimiento anual acumulativo media, estimada sobre intervalos de quince observaciones

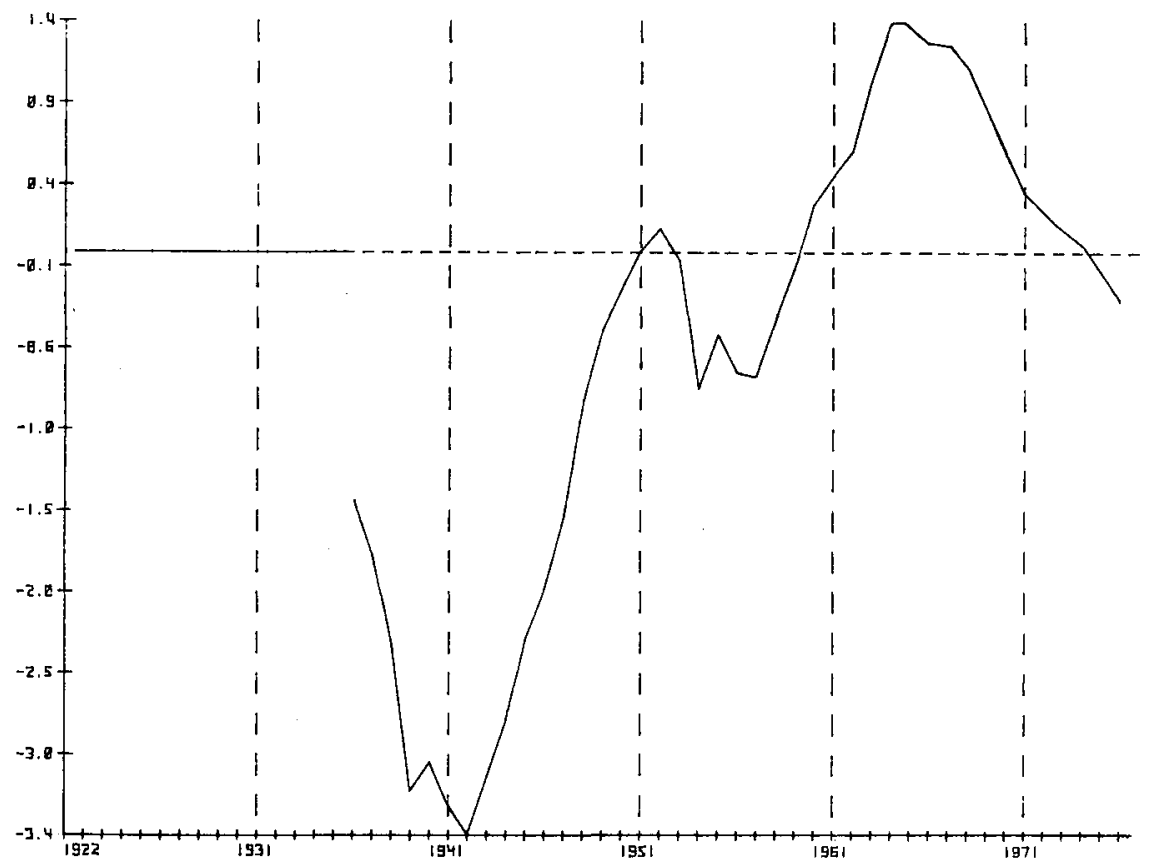

is A. SÁzz, o. c., gráfico 1 y cuadro 1, pp. 1008-1009.

16 Ibidem, p. 1012. 
Como ya hemos repetido, la fecundidad española baja suavemente desde 1965 y acelera su caída en los últimos tres años, en sincronía con los países occidentales. ¿Significaría esta sincronía que a partir de la subida observada desde la segunda mitad de los cincuenta, España se habría incorporado a esos ritmos profundos que rigen la dinámica de las poblaciones, en el régimen demográfico moderno de los países industriales occidentales?

Intentando identificar algún tipo de evolución cíclica de la fecundidad en este último período se describen en los gráficos 8.1 y 8.2 las desviaciones de la serie original de la tasa bruta de reproducción respecto a la curva de tendencia antes citada, $K(1+r)^{t}$, estimada sobre intervalos de 20 y 25 observaciones. No parece posible identificar ningún ciclo.

\section{GRAFICO NUM. 7.4}

Tasa de crecimiento anual acumulativo media, estimada sobre intervalos de veinte observaciones

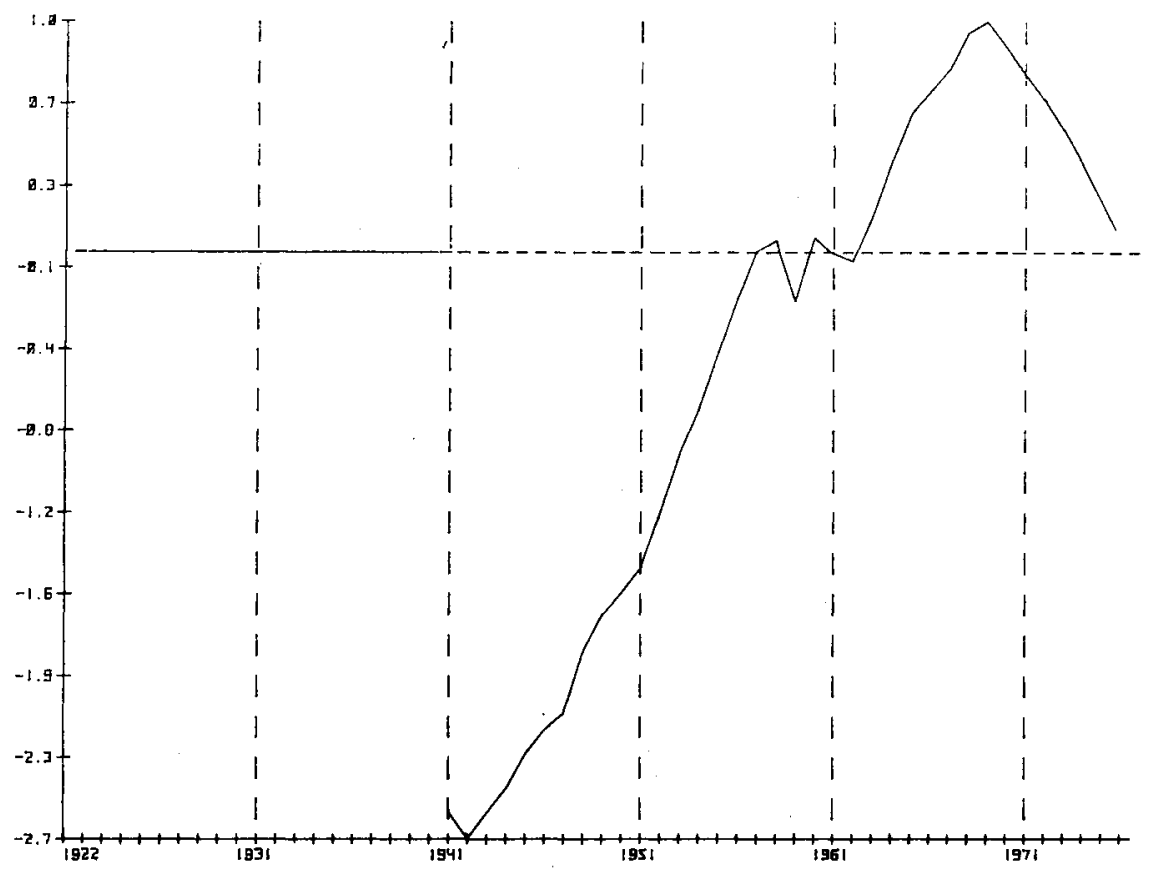

Aun conscientes de que no existe una causalidad directa de los determinantes económicos sobre la evolución de la fecundidad, hemos intentado verificar si existía en este último período alguna correlación entre la fecundidad y la actividad económica. En el gráfico 9 se describe la evolución del 
PIB desde 1955 a 1979. En la comparación de este gráfico con la tasa bruta de reproducción (gráfico 3) se observa que durante el período 1955-1958, de fecundidad creciente, se dan años de decrecimiento del PIB; sin embargo, la tasa bruta de reproducción sí parece acusar el impacto del plan de estabilización en los años siguientes y reacciona positivamente a la reactivación económica de la primera mitad de los sesenta. La evolución a la baja de la fecundidad desde 1965, en pleno auge económico, como en los demás países occidentales, demuestra una vez más la complejidad en la incidencia de las variables económicas sobre la fecundidad. ¿Podría explicarse entonces la caída de los tres últimos años porque una mayor posibilidad de control de la natalidad haría más sensible esta variable a las fluctuaciones económicas?

\section{GRAFICO NUM. 7.5}

Tasa de crecimiento anual acumulativo media, estimada sobre intervalos de veinticinco observaciones

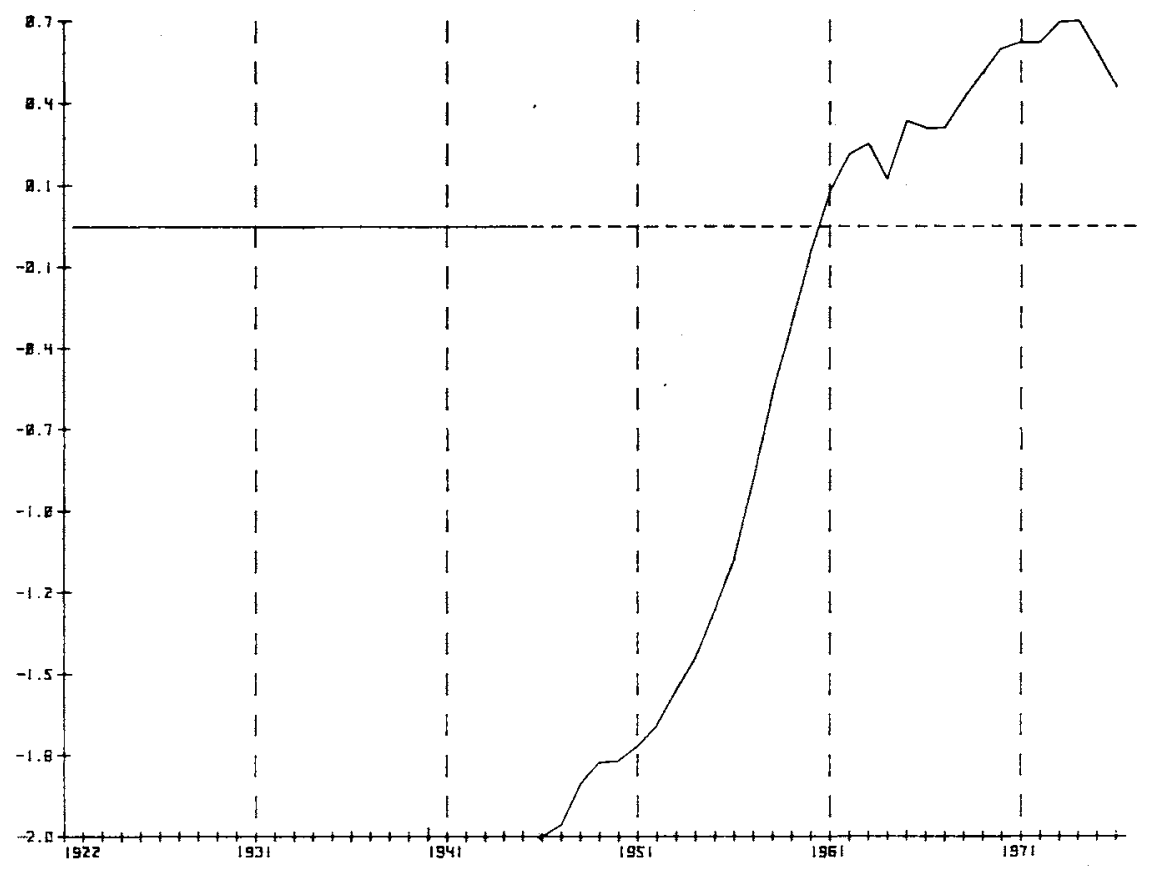

La tercera tesıs enunciada introduce la hipótesis de evolución cíclica de la fecundidad dentro del modelo de la transición demográfica. Siguiendo a J. Grauman, J. Bourgeois-Pichat ${ }^{17}$ afirma que las ideas de un individuo res17 J. Bourgeots-Pichat, o. c. 
pecto a la procreación se forman en la familia en que vive, de tal manera que las personas que han nacido y se han desarrollado en pequeñas familias serían más propensas a tener una descendencia numerosa. Se ha observado, en Francia, una correlación entre la descendencia final de las generaciones y la proporción de personas de esas generaciones que habían nacido en pequeñas familias. La subida de la natalidad observada hacia los años cuarenta coincidió con un aumento de la proporción de individuos procedentes de pequeñas familias y la caída de los quince últimos años ha comenzado cuando esta proporción ha disminuido. Los datos de Alemania Occidental, Inglaterra y Estados Unidos confirman la observación hecha en el caso francés.

\section{GRAFICO NUM. 8.1}

Desviaciones de la tasa bruta de reproducción respecto a la linea de tendencia $K(1+r)^{t}$ estimada sobre intervalos de veinte observaciones

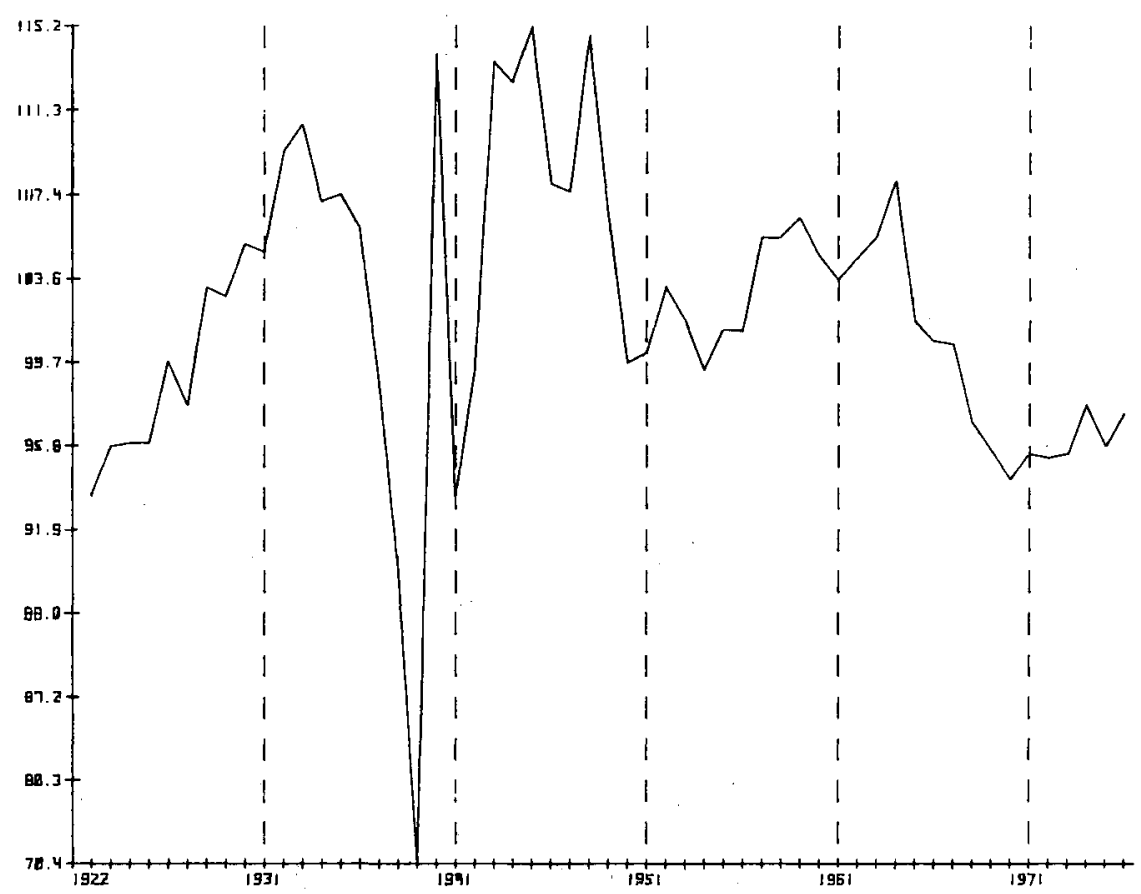

De hecho, la última fase del modelo de transición demográfica (baja fecundidad constante compensando más o menos una baja mortalidad también constante) no se ha observado nunca, y pertenece todavía al campo de la conjetura. 
Lo que se observa actualmente parece indicar que esta última fase no sería una fase de fecundidad constante, sino más bien una sucesión de oscilaciones producidas por las modificaciones en la estructura de las familias, resultantes a su vez de variaciones de la fecundidad. Como en el caso de Easterlin, la evolución favorable de la estructura de las familias en los paises occidentales citados permite anticipar un aumento de la fecundidad después de 1980. Así, pues, la evolución de la fecundidad en los próximos años constituirá un test decisivo para este modelo.

Está por realizar la aplicación de dicho modelo al caso español, tanto para explicar la coyuntura actual de fecundidad como su evolución futura.

\section{GRAFICO NUM. 8.2}

Desviación de la tasa bruta de reproducción respecto a la línea de tendencia $K(1+r)^{t}$ estimada sobre intervalos de veinticinco observaciones

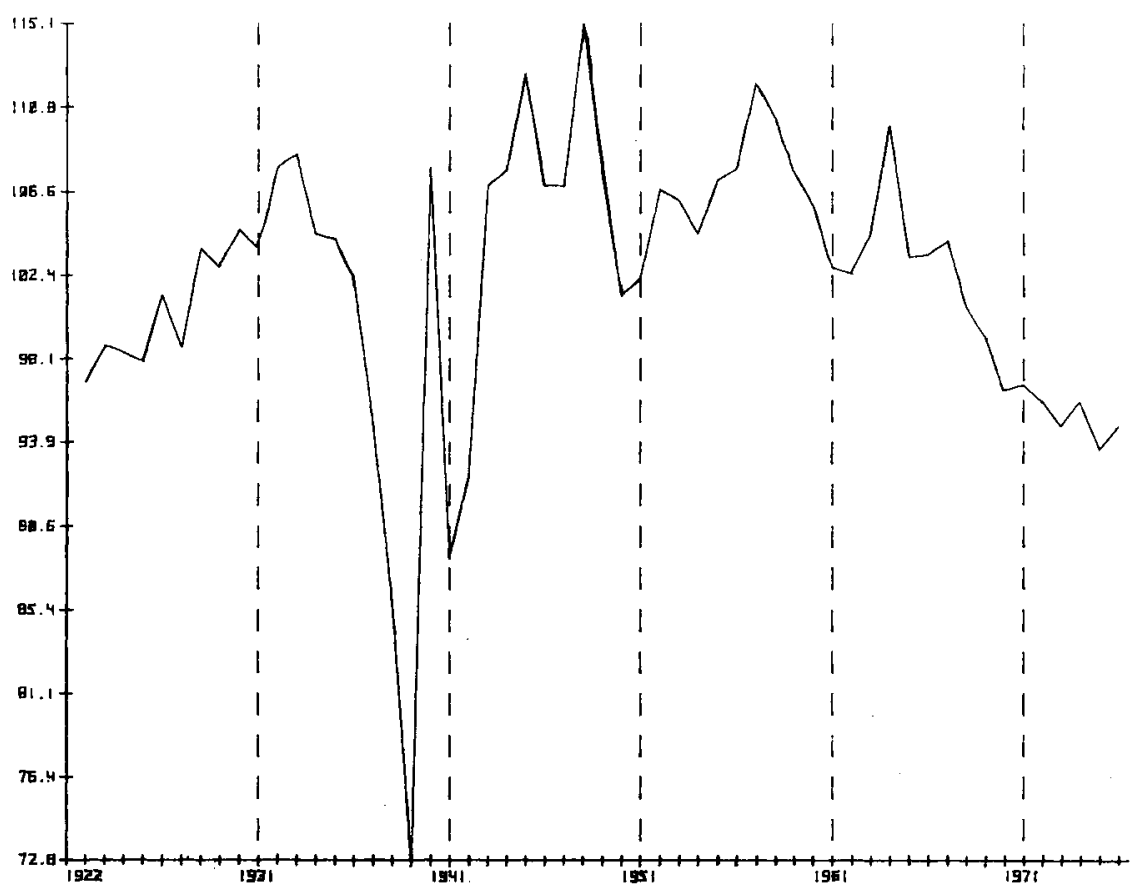




\section{GRAFICO NUM. 9}

Evolución de la producción y la inversión en los últimos veinticinco años Tasas de variación en pesetas constantes

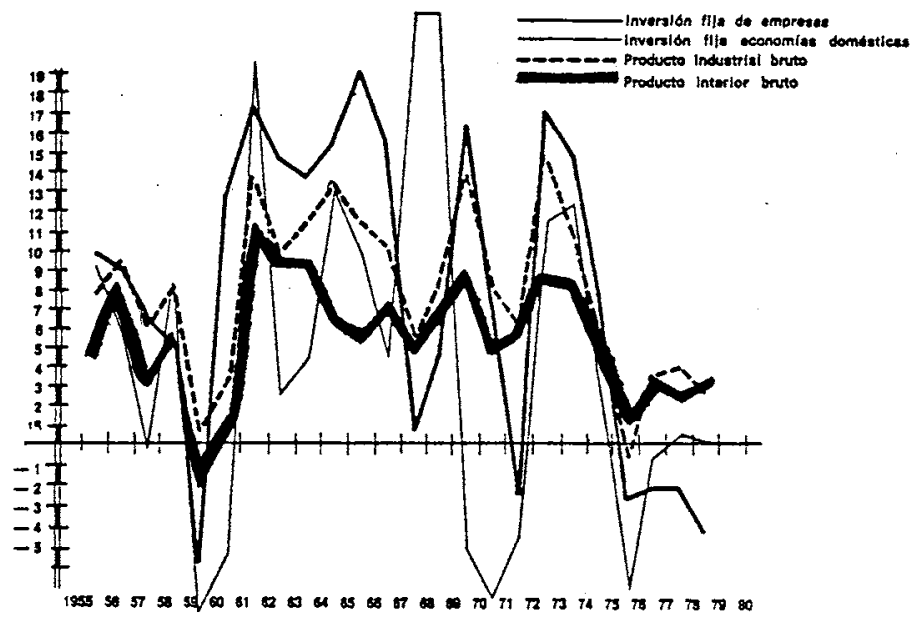

Fuente: INE, "La Renta Nacional en 1978 y su distribución", Madrid, 1978, pág. 48.

\section{La natalidad española a nivel provincial}

Como último intento para explicar la evolución reciente de la fecundidad en España, hemos pretendido verificar, con los datos disponibles, si se estaría produciendo una homologación en los comportamientos de procreación entre las regiones más natalistas y las más maltusianas, cuyo resultado final sería una caída en las tasas nacionales de fecundidad y natalidad.

A nivel provincial, el único indicador que ha sido posible utilizar es la tasa bruta de natalidad (TBN). En el cuadro VII se describe la evolución de este índice en el período 1960-1978*. En el cuadro VIII se presenta, para cada provincia, la diferencia entre la TBN de 1960 y la correspondiente a 1978 y en el cuadro 9 se ha clasificado a las provincias según los resultados obtenidos del cuadro 8. En el cuadro 10 se presenta el coeficiente de variación (desviación típica dividida por la tasa nacional) para cada una de las fechas del período estudiado.

Antes de exponer las conclusiones que se derivan del estudio de la información contenida en los cuadros 7 a 10 , es necesario analizar la calidad

- Las diferencias que se observan en la TBN recogida en este cuadro 7, correspondiente al total nacional con la serie que se presenta en el cuadro 1 , se deben a que las señas provinciales de la TBN no han sido corregidas para eliminar la falta de homogeneidad que supone la nueva definición de nacido vivo adoptada a partir de 1975, que incluye los muertos al nacer y los muertos antes de las 24 horas. La TBN nacional sin corregir del cuadro 7 es más adecuada para su comparación con las TBN provinciales, también sin corregir. 
CUADRO VII

Tasas de natalidad provinciales

\begin{tabular}{|c|c|c|c|c|c|c|c|c|c|c|}
\hline Provincias & & & 1960 & 1961 & 1962 & 1963 & 1964 & 1965 & 1966 & 1967 \\
\hline 1. Alava $\ldots$... & $\ldots$. & & 24,32 & 22.32 & 22.29 & 22,88 & 24,65 & 25,26 & 25,13 & 25.26 \\
\hline 2. Albacete $\ldots$... & $\ldots$. & $\ldots$ & 22,95 & 24,36 & 23,96 & 23,76 & 24,06 & 21.74 & 21,56 & 21,18 \\
\hline 3. Alicante $\ldots$... & $\ldots$. & $\ldots$ & 22,17 & 21,34 & 21.94 & 22.81 & 24.43 & 23.33 & 24,06 & 24,32 \\
\hline 4. Almería $\ldots$... . & $\ldots$. & $\ldots$ & 26,53 & 24,35 & 23,84 & 25.74 & 25,89 & 25,09 & 25,12 & 24.35 \\
\hline 5. Avila $\quad \ldots \quad \ldots \quad \ldots$. & ... & $\ldots$ & 18,36 & 19,28 & 19,11 & 17.90 & 17,00 & 15,89 & 14,76 & 14.25 \\
\hline 6. Badajoz $\ldots \ldots$. & $\ldots$. & $\ldots$ & 21,61 & 22,21 & 21,79 & 20,91 & 20,59 & 18,20 & 17,19 & 16,48 \\
\hline 7. Baleares ... ... & $\ldots$. & $\ldots$ & 17,21 & 16,99 & 17,73 & 19.26 & 20,75 & 20.69 & 21,48 & 22,29 \\
\hline 8. Barcelona ... ... & $\ldots$. & $\ldots$ & 20,45 & 18,19 & 19,08 & 19,96 & 21.50 & 21,52 & 21,71 & 22,22 \\
\hline 9. Burgos $\ldots \ldots \ldots$ & $\ldots$. & $\ldots$ & 18,94 & 19,37 & 18.79 & 18,63 & 17.91 & 16,93 & 15,96 & 15,88 \\
\hline 10. Cáceres $\ldots \ldots \ldots \ldots$. & $\ldots$ & $\ldots$ & 22,29 & 22,84 & 22,57 & 21,69 & 21,05 & 19,00 & 18 & 16,70 \\
\hline 11. Cádiz $\ldots \ldots \ldots$. & $\ldots$. & $\ldots$ & 26,57 & 25,96 & 25,39 & 25.94 & 27,11 & 25,86 & 25.19 & 25,60 \\
\hline 12. Castellón $\ldots \ldots$. & $\ldots$. & $\ldots$ & 16,92 & 16,30 & 16.86 & 17,64 & 17,56 & 18,08 & 18,47 & 19,13 \\
\hline 13. Ciudad Real ... & $\ldots$. & $\ldots$ & 24,09 & 23,95 & 22,20 & 21,71 & 21,94 & 19,81 & 18,16 & 17,80 \\
\hline 14. Córdoba ... $\ldots \ldots$ & $\ldots$. & $\ldots$ & 24,32 & 24,03 & 22,97 & 23,41 & 24,05 & 21,47 & 20,48 & 20,34 \\
\hline 15. Coruña (La) ... & $\ldots$. & $\ldots$ & 17.89 & 18,16 & 17,60 & 17,69 & 17,72 & 17,25 & 17,28 & 17,35 \\
\hline 16. Cuenca $\ldots \ldots \ldots$ & $\ldots$. & $\ldots$ & 21,16 & 21,31 & 20,19 & 19,24 & 17,85 & 16.10 & 15,16 & 14,59 \\
\hline 17. Gerona ... ... $\ldots$ & $\ldots$. & $\ldots$ & 16,90 & 16,08 & 16,76 & 17,91 & 17,89 & 17,99 & 18,03 & 17,80 \\
\hline 18. Granada ....... & $\ldots$. & $\ldots$ & 24,92 & 26,05 & 25,37 & 25,38 & 26,56 & 24,74 & 23.70 & 23,35 \\
\hline 19. Guadalajara ... . & $\ldots$ & $\ldots$ & 16,32 & 16,70 & 16,30 & 15,75 & 15,17 & 13,79 & 12,58 & 12,48 \\
\hline 20. Guipúzcoa $\ldots \ldots$ & $\ldots$ & $\ldots$ & 26,67 & 23,66 & 24,27 & 24,85 & 25,66 & 25,37 & 24,40 & 23,68 \\
\hline 21. Huelva $\ldots \ldots \ldots$ & $\ldots$ & $\ldots$ & 23,20 & 20,79 & 20,03 & 20,58 & 20,59 & 19,22 & 19,36 & 19,91 \\
\hline 22. Huesca $\ldots . \ldots$. & . & & 15.34 & 15,61 & 15.38 & 14,87 & 14,76 & 14.37 & 13,61 & 13.12 \\
\hline 23. Jaén $\quad .$. & $\ldots$ & $\ldots$ & 25,16 & 25,92 & 24,91 & 25,28 & 26,47 & 23,52 & 20,74 & 21,94 \\
\hline 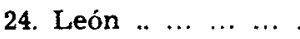 & $\ldots$ & $\ldots$ & 21,76 & 21,35 & 20.58 & 19,87 & 19,23 & 17,77 & 16,85 & 15,99 \\
\hline 25. Lérida $\ldots \ldots^{\ldots} \quad \ldots \ldots$. & $\ldots$ & $\ldots$ & 16,81 & 18,58 & 18,17 & 17,81 & 18,75 & 17,98 & 17,44 & 16,99 \\
\hline 26. Logroño .. ... ... & $\ldots$ & $\cdots$ & 18,12 & 18,71 & 18,34 & 17,92 & 18,67 & 17,50 & 16,87 & 16,93 \\
\hline 27. Lugo $\ldots \ldots \ldots c$ & $\ldots$ & $\ldots$ & 15,57 & 16,23 & 15,79 & 14,50 & 14,27 & 13,59 & 13,23 & 12,87 \\
\hline 28. Madrid $\ldots \ldots \ldots$ & $\ldots$ & & 25,59 & 22,05 & 23,13 & 23,22 & 24,80 & 24,58 & 24,26 & 24,86 \\
\hline 29. Málaga $\ldots \ldots \ldots$ & $\ldots$ & $\ldots$ & 20,30 & 21,52 & 21,49 & 23,18 & 25,17 & 23,95 & 23,86 & 24,39 \\
\hline 30. Murcia ... ...... & $\ldots$ & $\ldots$ & 24,00 & 23,42 & 24,97 & 26.17 & 25,67 & 25,01 & 24,68 & 24,75 \\
\hline 31. Navarra ....... & $\ldots$ & $\ldots$ & 19,81 & 20,04 & 19,67 & 20,42 & 20,72 & 20,24 & 20,13 & 20,34 \\
\hline $\begin{array}{lllll}\text { 32. Orense } & \ldots & \ldots & \ldots\end{array}$ & $\ldots$ & $\ldots$ & 14,84 & 15,12 & 14,52 & 14,29 & 14,32 & 13,82 & 13,39 & 13,47 \\
\hline 33. Oviedo $\ldots \ldots \ldots$ & . & . & 20,70 & 18,70 & 18,03 & 17,31 & 16,95 & 16,15 & 15,40 & 16,04 \\
\hline 34. Palencia .. ... ... & $\cdots$ & $\ldots$ & 19,85 & 20,51 & 19,86 & 18,65 & 15,50 & 16,97 & 15,63 & 14,85 \\
\hline 35. Palmas (Las) ... & $\ldots$ & $\ldots$ & 29,59 & 29,28 & 29,71 & 30,28 & 31,48 & 30,81 & 29,88 & 30,45 \\
\hline 36. Pontevedra .. ... & $\ldots$ & $\ldots$ & 21,03 & 21,52 & 20,83 & 20,94 & 21,00 & 21,07 & 21,73 & 21,94 \\
\hline 37. Salamanca $\ldots \ldots$ & $\ldots$ & $\ldots$ & 20,83 & 20,72 & 20,41 & 20,12 & 19,30 & 18,23 & 17,44 & 17,28 \\
\hline 38. S. C. de Tenerife & e ... & $\ldots$ & 23,14 & 23,66 & 23,63 & 24,44 & 25,17 & 24,25 & 23,75 & 23,41 \\
\hline 39. Santander $\ldots \ldots$ & $\cdots$ & $\ldots$ & 22,22 & 20,93 & 20,29 & 20,18 & 19,74 & 19,63 & 19,74 & 19,80 \\
\hline 40. Segovia ... ...... & $\ldots$ & $\ldots$ & 18,58 & 19,93 & 19,13 & 18,73 & 18,50 & 16,87 & 16,26 & 15,85 \\
\hline 41. Sevilla $\ldots \ldots$... & $\ldots$ & $\ldots$ & 23,96 & 23,60 & 23,62 & 24,64 & 25,82 & 24,45 & 23,88 & 24,25 \\
\hline 42. Soria $. . . \ldots . . .$. & $\ldots$ & $\ldots$ & 16,46 & 16,29 & 16,23 & 15,51 & 14,88 & 13,69 & 12,44 & 12,52 \\
\hline 43. Tarragona $\ldots \ldots$ & . & & 16,37 & 16,89 & 18,19 & 18,03 & 19,48 & 19,38 & 20,55 & 20,69 \\
\hline 44. Teruel $\ldots$. $\ldots$... & $\ldots$ & & 15,52 & 16,01 & 15,67 & 15,69 & 14.78 & 13,84 & 12,98 & 12,16 \\
\hline 45. Toledo $\ldots . . . .$. & $\cdots$ & $\cdots$ & 19,23 & 20,03 & 19,60 & 19,04 & 18,26 & 16,81 & 15,72 & 15,55 \\
\hline 46. Valencia .. ... ... & $\cdots$ & 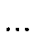 & 19,47 & 19,50 & 19,82 & 20,50 & 21,93 & 21,75 & 22,14 & 22,72 \\
\hline 47. Valladolid $\ldots \ldots$ & . & $\ldots$ & 22,31 & 21,46 & 21,12 & 21,13 & 21,16 & 21,14 & 21,12 & 21.47 \\
\hline 48. Vizcaya $\ldots \ldots \ldots$ & $\ldots$ & ... & 28,63 & 23,67 & 24,43 & 24,99 & 25,86 & 25,09 & 24,54 & 24,00 \\
\hline 49. Zamora $\ldots \ldots \ldots$ & & & 18,50 & 18,93 & 18,35 & 17,10 & 17,04 & 15,32 & 14,23 & 14,13 \\
\hline 50. Zaragoza $\ldots$... & & & 19,56 & 19,06 & 19,59 & 19,24 & 20,03 & 18,99 & 19,02 & 19,12 \\
\hline Nacional ........ & & $\ldots$ & 21,73 & 21,13 & 21,00 & 21,32 & 21,98 & 21,13 & 20,76 & 20,91 \\
\hline
\end{tabular}




\begin{tabular}{|c|c|c|c|c|c|c|c|c|c|c|}
\hline 1968 & 1969 & 1970 & 1971 & 1972 & 1973 & 1974 & 1975 & 1976 & 1977 & 1978 \\
\hline 21,01 & 20,84 & 20,44 & 20,78 & 20,19 & 19,82 & 19.82 & 18,61 & 17,95 & 18,72 & 16,35 \\
\hline 22.42 & 22,83 & 20.26 & 19,90 & 19,58 & 19,26 & 19,31 & 19,95 & 19,80 & 17,32 & 18,18 \\
\hline 22.13 & 22.08 & 21,33 & 21,17 & 20,51 & 20,95 & 21,19 & 18,61 & 20,31 & 19,61 & 18,09 \\
\hline 23,61 & 23,25 & 21,58 & 21,54 & 21,39 & 21,22 & 20,68 & 20,43 & 21,62 & 20,51 & 19,72 \\
\hline 14,72 & 13,85 & 13,72 & 12,87 & 12,89 & 12,75 & 12,42 & 12,18 & 11,21 & 12,09 & 11,64 \\
\hline 18,62 & 18.71 & 17,92 & 17,60 & 17,41 & 17,09 & 16,87 & 16,63 & 17,03 & 15,80 & 16,48 \\
\hline 19,84 & 20,33 & 19,44 & 20.85 & 20.51 & 20,70 & 20,53 & 18,40 & 17,46 & 17,02 & 15,82 \\
\hline 20,73 & 20,30 & 20,38 & 20,60 & 20,58 & 20,48 & 20,02 & 19,96 & 16,27 & 18,06 & 16,24 \\
\hline 17,13 & 16.83 & 15.53 & 15,89 & 15,60 & 15,65 & 15,87 & 15,84 & 17,31 & 15,57 & 14,72 \\
\hline 18,35 & 17,43 & 16,15 & 15.64 & 15,34 & 15,15 & 14,28 & 13,87 & 14,58 & 13,85 & 13,85 \\
\hline 25.46 & 25.46 & 25,52 & 25,04 & 24,54 & 23,95 & 24,25 & 23,71 & 24,26 & 23,34 & 22,19 \\
\hline 17.27 & 16.50 & 15,78 & 16,48 & 16,39 & 17,04 & 17,14 & 16,40 & 17,15 & 17,00 & 15,98 \\
\hline 19.17 & 18,49 & 17,60 & 16,50 & 16,86 & 16,55 & 16,34 & 15,97 . & 16,13 & 15,30 & 15,55 \\
\hline 20,61 & 20,23 & 19,69 & 19,05 & 18,90 & 18,44 & 18,67 & 18,62 & 19,02 & 17,69 & 17,76 \\
\hline 17.67 & 17,24 & 17,20 & 17,45 & 17.94 & 17,80 & 18,22 & 18.12 & 17,95 & 17,09 & 16,83 \\
\hline 16.88 & 15.65 & 14,61 & 13,70 & 13.00 & 13,29 & 12,82 & 12,21 & 12.74 & 12,55 & 11,97 \\
\hline 16,26 & 16.47 & 16.31 & 16,82 & 16,77 & 16.62 & 17,20 & 16,88 & 16,84 & 17,58 & 16,52 \\
\hline 23.40 & 22,62 & 20,87 & 20,69 & 20,09 & 19,91 & 20,07 & 19,34 & 19,68 & 18,21 & 18,58 \\
\hline 12.71 & 12,56 & 12.18 & 11,85 & 12,95 & 12,07 & 12,97 & 12,63 & 13,80 & 13,98 & 13,11 \\
\hline 21,24 & 21,10 & 21,07 & 20,41 & 20,24 & 19,42 & 18,37 & 17,25 & 17,62 & 17,98 & 15,08 \\
\hline 20.95 & 20,26 & 19,40 & 19,30 & 19,39 & 18,86 & 19,12 & 19,71 & 18,71 & 19,50 & 19,12 \\
\hline 11,96 & 12,21 & 11,60 & 11,51 & 11,67 & 11,35 & 11,17 & 11,73 & 11,90 & 13,28 & 11,57 \\
\hline 21,58 & 20,34 & 19,67 & 18,37 & 18,60 & 17,92 & 17,46 & 16,83 & 17,57 & 16,15 & 16,08 \\
\hline 16.77 & 16.62 & 15.68 & 15,49 & 15,12 & 14,38 & 14,64 & 13.43 & 14,32 & 13,70 & 13,95 \\
\hline 16,36 & 16,33 & 15.94 & 15,33 & 15,36 & 15,26 & 15,82 & 15,39 & 16,20 & 14,67 & 15,17 \\
\hline 16,75 & 16,64 & 15,95 & 16,18 & 16,28 & 16,16 & 16,42 & 16,88 & 17,17 & 15,73 & 16,31 \\
\hline 13,28 & 12,92 & 12,73 & 12,38 & 12,35 & 12,44 & 12,85 & 12,36 & 12,92 & 11,69 & 11,78 \\
\hline 22,74 & 22,65 & 22,24 & 22.52 & 21,96 & 21,42 & 21,90 & 20,68 & 20,38 & 19,85 & 18,56 \\
\hline 23.23 & 23,42 & 21.51 & 21.85 & 21,77 & 22,08 & 22,07 & 21,15 & 20,92 & 20,20 & 19,66 \\
\hline 24,58 & 24,19 & 23,43 & 23,47 & 23,33 & 23,48 & 24,24 & 22,08 & 22,16 & 21,48 & 21,39 \\
\hline 19,41 & 19,59 & 18,32 & 18,44 & 17,85 & 17,35 & 17,37 & 16,75 & 17,38 & 17,11 & 15,85 \\
\hline 13,66 & 12,91 & 12,25 & 12,04 & 11,93 & 11,65 & 12,54 & 11,91 & 12,27 & 10,64 & 10,95 \\
\hline 16,01 & 16,19 & 15.75 & 16,52 & 16,51 & 16,16 & 16,82 & 16,03 & 16,69 & 15,67 & 14,64 \\
\hline 15,44 & 14,81 & 14.55 & 14,08 & 13,60 & 13,65 & 13,32 & 13,23 & 13,91 & 13,30 & 14,20 \\
\hline 27,63 & 27,90 & 28,18 & 27,89 & 27,00 & 26,44 & 25,01 & 21,25 & 21,97 & 21,03 & 19,99 \\
\hline 21,06 & 20,76 & 20,07 & 19,69 & 19,85 & 19,56 & 20,40 & 20,75 & 21,27 & 19,09 & 18,35 \\
\hline 18,20 & 18,12 & 16,53 & 16,44 & 15,63 & 15,25 & 14,85 & 14,36 & 14,99 & 13,39 & 14,70 \\
\hline 21,37 & 21,51 & 22,11 & 22,50 & 21,17 & 22,06 & 22,02 & 20,11 & 20,09 & 18,05 & 17,01 \\
\hline 18,97 & 18,98 & 18,42 & 17,63 & 17,64 & 17,99 & 17,84 & 18,25 & 18,77 & 17,80 & 17,48 \\
\hline 15.87 & 15,94 & 15,60 & 15,46 & 15,19 & 14,38 & 14,02 & 14,21 & 14,26 & 14,17 & 13,68 \\
\hline 22,63 & 22,35 & 23,46 & 23,58 & 23,26 & 23,23 & 23,72 & 22,37 & 23,53 & 22,74 & 22,24 \\
\hline 12,47 & 12,94 & 11,74 & 11,73 & 11,55 & 11,00 & 11,04 & 10.45 & 11,25 & 12,12 & 10,78 \\
\hline 17,70 & 18,09 & 16,87 & 17,49 & 17,59 & 17,85 & 18,75 & 18,34 & 19,28 & 18,29 & 17,17 \\
\hline 13,27 & 12,56 & 11,71 & 10,94 & 10,97 & 10,67 & 10,85 & 10,83 & 10,81 & 12,44 & 10,98 \\
\hline 17,31 & 16,68 & 15,63 & 15,25 & 15,24 & 15,07 & 15,52 & 15,59 & 16,52 & 15,96 & 15,71 \\
\hline 20,35 & 20,08 & 19,15 & 19,00 & 19,14 & 19,10 & 19,80 & 19,46 & 19,48 & 19,19 & 18,60 \\
\hline 21,06 & 20,97 & 19,46 & 19,04 & 19,56 & 19,68 & 20,84 & 18,77 & 20,47 & 18,33 & 18,19 \\
\hline 21,46 & 20,87 & 20,86 & 21,35 & 20,89 & 20,01 & 19,54 & 18,64 & 18,89 & 18,42 & 16,96 \\
\hline 14,75 & 14,55 & 13,53 & 13,28 & 12,57 & 12,23 & 11,80 & 12,09 & 12,51 & 12,46 & 12,10 \\
\hline 17,66 & 17,36 & 16,75 & 17,04 & 17,27 & 16,76 & 16,86 & 17,06 & 16,79 & 15,63 & 14,99 \\
\hline 20,22 & 20,00 & 19,50 & 19,55 & 19,37 & 19,19 & 19,43 & 18,64 & 18,47 & 18,05 & 17,21 \\
\hline
\end{tabular}


de los indicadores utilizados. Es sabido que la TBN no elimina el efecto de la estructura por edad al incluir en el denominador los efectivos totales, no los efectivos en edad fecunda. Por otra parte, respecto al cuadro 8 , se tomaron los valores de la TBN en 1960 y en 1978, a pesar de que el decrecimiento en el tiempo de la TBN no es continuo, porque los valores máximos de la tasa no se producen en las mismas fechas en todas las provincias, lo que introducía un elemento de arbitrariedad. La diferencia entre las tasas al principio y al final del período tiene la ventaja de expresar un balance de lo ocurrido en el mismo intervalo temporal para todas las provincias.

\section{CUADRO VIII}

\section{Diferencia entre la TBN en 1960 y 1978}

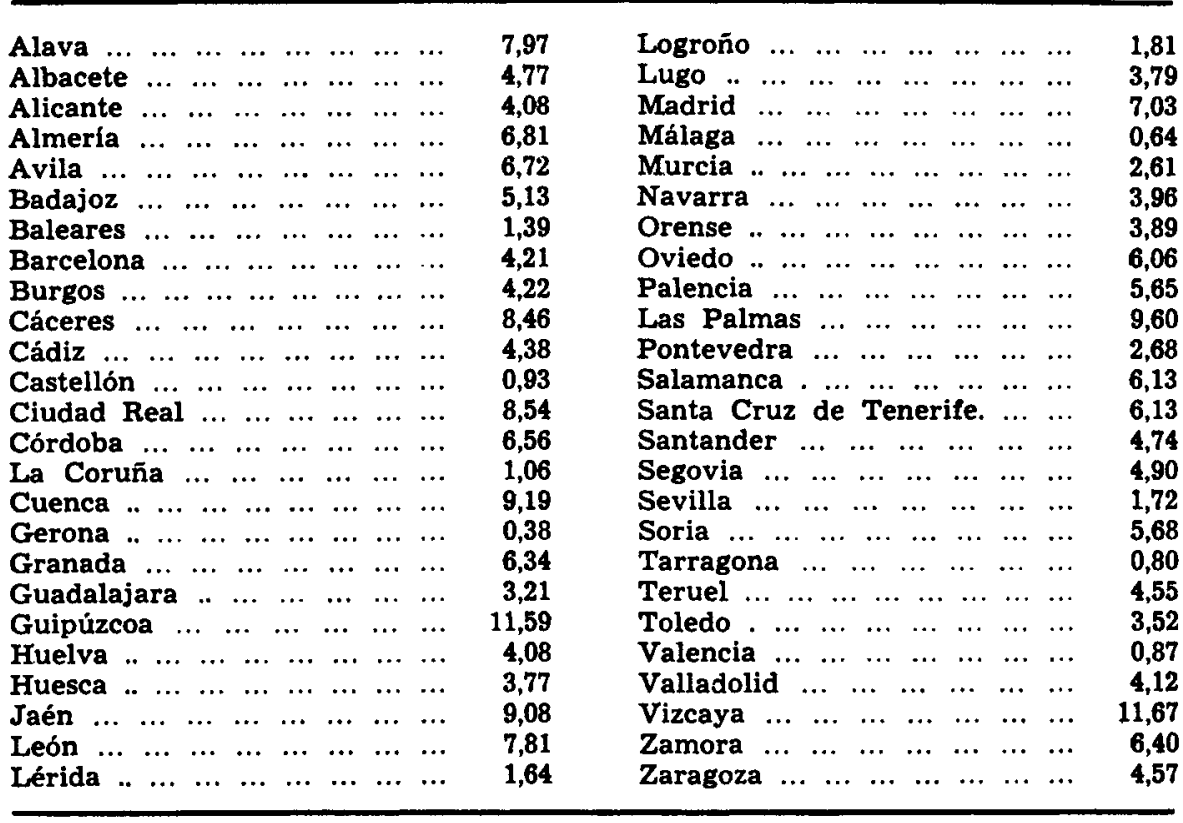

El coeficiente de variación que se recoge en el cuadro $\mathrm{X}$ ha sido calculado sin ponderar las tasas provinciales por los efectivos en cada provincia.

Con todas las limitaciones impuestas por la calidad de la información que se maneja, los resultados del cuadro $X$ muestran que la dispersión de las tasas provinciales respecto del promedio nacional sigue una evolución irregular en el tiempo; a partir de 1973 se detecta, sin embargo, un descenso en el coeficiente de variación que indica que las tasas de natalidad tienden a reducir sus diferencias, apuntando hacia una mayor homogeneidad. 
De la observación del cuadro IX se puede deducir la forma en que dicha homogeneización se ha producido.

En efecto, Madrid y las tres provincias vascas, focos receptores de migraciones, se sitúan entre las provincias cuya TBN está por encima de la media nacional (véase cuadro VII) y, al mismo tiempo, entre las provincias cuya natalidad ha caído más intensamente en el periodo estudiado (véase cuadro IX). La adaptación de los emigrados al régimen de fecundidad de las zonas receptoras ha sido un factor a tener en cuenta en el descenso de la natalidad a nivel nacional.

\section{CUADRO IX}

Clasificación de las provincias según la diferencia de la TBN en 1960 y 1978

Diferencia superior

a 7 puntos por mil
Alava, Guipúzcoa, Vizcaya.

Madrid

Cuenca, Ciudad Real Cáceres, León

Las Palmas

Jaén

Almerfa, Granada, Córdoba

Avila, Badajoz, Palencia, Salamanca, Soria,

Zamora

Oviedo

Santa Cruz de Tenerife

Cádiz, Huelva

Murcia, Albacete, Alicante

Navarra, Zaragoza, Huesca, Teruel

Orense, Lugo, Pontevedra

Guadalajara, Santander, Segovia, Toledo, Valladolid, Burgos

Barcelona

Diferencia inferior

Baleares

Castellón, Valencia

La Coruña

Gerona, Lérida, Tarragona

Logroño

Sevilla, Málaga

También el fenómeno migratorio explica la caída en la TBN que se observa en provincias como Cuenca, Ciudad Real, Cáceres, León y Jaén, provincias 
envejecidas en su estructura por edades al ser centros emisores de migraciones.

Se observa también en el cuadro IX que en el intervalo 7.5 se sirúan provincias como Almería, Granada y Córdoba, con TBN superiores a la tasa nacional, y también Avila, Badajoz, Palencia, Salamanca, Soria y Zamora, todas ellas provincias con TBN por debajo de la media nacional.

La misma observación puede hacerse si se estudian las provincias que en el cuadro IX se sitúan en el intervalo 4.99-2.

De todo ello cabe deducir que durante el periodo de observación, en su conjunto, no se ha producido una disminución sensible del comportamiento de procreación diferencial de las provincias y que el acercamiento que se detecta entre las tasas de natalidad provinciales a partir de 1973 se explica, sobre todo, por la interferencia del fenómeno migratorio, que ha provocado un envejecimiento de las zonas emisoras de emigración y un rejuvenecimiento en las zonas receptoras, en las que parece haberse producido una adaptación posterior de los emigrados a las pautas de fecundidad del lugar de destino.

\section{CUADRO X}

Coeficiente de variación interprovincial de la TBN 1960-1978

(Desviación típica dividida por la tasa nacional)

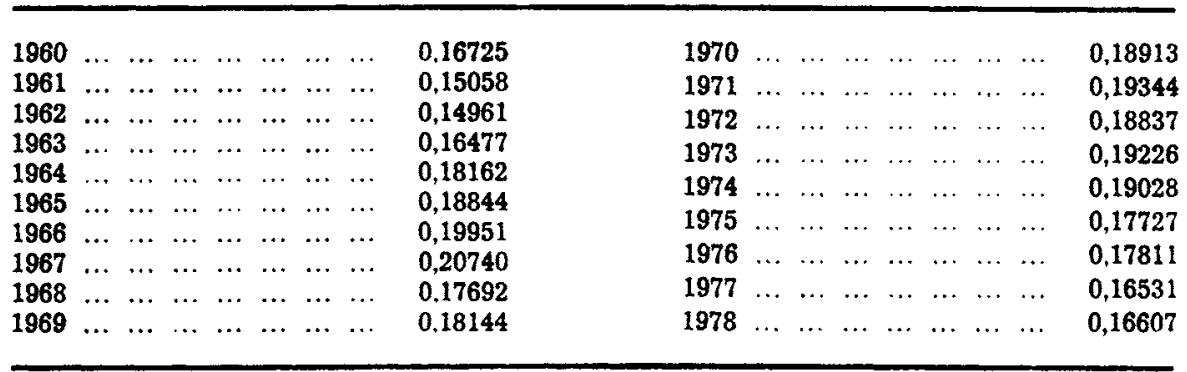

\section{BIBLIOGRAFIA}

J. N. Branaen: "L'Europe: Données statistiques", Population, núms. 4-5, 1978.

J. Bourgeors Pichat: "La baisse actuelle de la fécondité en Europe s'inscrit-elle dans le modèle de la transition démographique", Population, núm. 2, 1979.

J. C. Chenais: "L'effet multiplicatif de la transition démographique", Population, número 6, 1979.

R. EASTERLIN y otros: "Demographic influences on economic stability: The United States experience", Population and Development Review, marzo 1978.

M. Fresez-Losey: "Le colloque de Lille: la baisse de la fécondité", Population, número 6, 1979.

A. MonNien: "La conjoncture démographique: L'Europe et les pays développés d'Outre-Mer", Population, núms. 4-5, 1979.

OCDE, L'évolution démographique de 1950 à 1990, París, 1979.

A. Sízz, "La fécondité en Espagne depuis le début du siècle", Population, número $6,1979$.

Ch. F. Westofr, "Matrimonio y fertilidad en los paises desarrollados". Scientific American Rev., edición española, febrero 1979. 